\title{
OPTICAL SPECTROSCOPY AND X-RAY DETECTIONS OF A SAMPLE OF QUASARS AND ACTIVE GALACTIC NUCLEI SELECTED IN THE MID-INFRARED FROM TWO SPITZER SPACE TELESCOPE WIDE-AREA SURVEYS
}

\author{
M. Lacy, ${ }^{1}$ A. O. Petric, ${ }^{1,2}$ A. Sajina, ${ }^{1}$ G. Canalizo, ${ }^{3}$ L. J. Storrie-Lombardi, ${ }^{1}$ \\ L. Armus, ${ }^{1}$ D. Fadda, ${ }^{1}$ and F. R. Marleau ${ }^{1}$ \\ Received 2006 April 24; accepted 2006 September 19
}

\begin{abstract}
We present optical spectroscopy of a sample of 77 luminous active galactic nuclei (AGNs) and quasars selected on the basis of their mid-infrared colors. Our objects are selected from the Spitzer Extragalactic First Look Survey and the SWIRE XMM-Newton Large-Scale Structure Survey (XMM-LSS) fields, with a typical $24 \mu \mathrm{m}$ flux density of $5 \mathrm{mJy}$. The median redshift is 0.6 , with a range of $\sim 0.05-4$. Only $33 \%$ ( 25 out of 77 ) of these objects are normal type 1 quasars, with no obscuration. Forty-four percent (34 out of 77) are type 2 objects, with high-ionization, narrow emission lines, and 14\% (11 out of 77) are dust-reddened type 1 quasars, showing broad lines but a dust-reddened or unusually weak quasar continuum. Nine percent (7 out of 77) show no sign of an AGN in the optical spectrum, having either starburst spectra or spectra that could be of either starburst or LINER type. These latter objects are analogous to the X-ray-detected population of AGNs with weak or nonexistent optical AGN emission (the X-ray-bright, optically normal galaxies). Of our objects from the SWIRE field, 21 fall within moderately deep XMM-Newton exposures. All the unobscured quasars and about half the obscured quasars are detected in these exposures. This sample, when taken together with other samples of Spitzer-selected AGNs and quasars and results from X-ray studies, confirms that obscured AGNs dominate the AGN and quasar number counts of all rapidly accreting supermassive black hole systems, at least for $z \lesssim 4$. This implies a high radiative efficiency for the black hole accretion process.
\end{abstract}

Key words: galaxies: Seyfert — infrared: galaxies — quasars: general

\section{INTRODUCTION}

Quasars whose optical emission is hidden by dust in the optical and gas in the soft X-ray have always been hard to find. However, a large type 2 (hidden) quasar population has long been predicted, both from the statistics of Seyfert 1 and 2 galaxies and from those of radio galaxies and radio-loud quasars. A large population of hidden active galactic nuclei (AGNs) is also predicted from models of the X-ray background (e.g., Comastri et al. 1995; Worsley et al. 2005), although most models predict that these AGNs are of Seyfert rather than quasar luminosity. Some success in finding dust-obscured quasars has been obtained using selection based on the Two Micron All Sky Survey (2MASS) to find lightly dustreddened (rest-frame extinctions $A_{V} \sim 2$ ) quasars, based either on 2MASS colors alone (Cutri et al. 2001) or on 2MASS combined with a radio detection and very red optical-infrared colors (Glikman et al. 2004). Only recently, however, have large samples of type 2 quasars, with rest-frame $A_{V} \gtrsim 5$, been found. Zakamska et al. (2003) used the Sloan Digital Sky Survey (SDSS) to find objects with the high-ionization narrow-line spectra characteristic of type 2 AGNs. Combining Infrared Space Observatory (ISO) and 2MASS data allowed Haas et al. (2004a) and Leipski et al. (2005) to find significant numbers of obscured AGNs, including several type 2 quasars. Spitzer Space Telescope data have been used to find highly obscured AGNs, both through midinfrared color selection (Lacy et al. 2004 [hereafter Paper I], 2005a; Stern et al. 2005; Alonso-Herrero et al. 2006; Polletta et al. 2006) and joint radio-infrared selection (Martínez-Sansigre et al. 2005, 2006; Weedman et al. 2006; Donley et al. 2005). Type 1 quasars

\footnotetext{
${ }^{1}$ Spitzer Science Center, California Institute of Technology, Pasadena, CA, USA; mlacy@ipac.caltech.edu, sajina@ipac.caltech.edu, lisa@ipac.caltech.edu.

${ }^{2}$ Columbia University, New York, NY, USA.

${ }^{3}$ University of California, Riverside, CA, USA.
}

selected through joint Spitzer and optical colors and/or morphologies have been targeted by several groups (Brown et al. 2006; Hatziminaoglou et al. 2005; Siana et al. 2007). Also, the midinfrared properties of X-ray-selected AGNs have been discussed by Treister et al. (2004) and Barmby et al. (2006).

Mid-infrared selection of AGNs is effective because dust heated by the AGN forms a "calorimeter" whose luminosity is not as strongly affected by obscuration as that in most other wavelength bands (except the radio; e.g., Spinoglio et al. 1995; Meisenheimer et al. 2001; Ogle et al. 2006). Richards et al. (2006) show that the bolometric correction in the mid-infrared varies little for different subsamples of type 1 quasars (e.g., optically or infrared luminous, optically red or optically blue) or luminosity, and that about $15 \%$ of the bolometric luminosity of a quasar is emitted at mid-infrared wavelengths. Integration over the Richards et al. (2006) mean quasar SED as a whole suggests that about $40 \%$ of the emission from a typical quasar is reprocessed by dust in the infrared. This means that mid-infrared selection of AGNs is probably a good proxy for selection on the basis of bolometric luminosity, at least for those objects that are not so highly obscured that their midinfrared luminosity is significantly suppressed. Much of the dust emission is thought to arise in the "obscuring torus" required by unified schemes (e.g., Antonucci 1993), although it is becoming increasingly clear that host dust in the narrow-line region of AGNs also contributes significantly (e.g., Mason et al. 2006). AGNs are an important global contributor to the mid-infrared luminosity density. Brand et al. (2006) show that the luminosity density of mid-infrared emission in samples of MIPS $24 \mu \mathrm{m}$ sources selected at millijansky levels is dominated by AGNs; only at submillijansky levels do star-forming galaxies begin to dominate.

In this paper we focus on optical spectroscopic follow-up of AGN candidates selected according to the mid-infrared color criteria presented in Paper I. By selecting relatively bright objects from wide-area Spitzer surveys we are able to find many obscured 


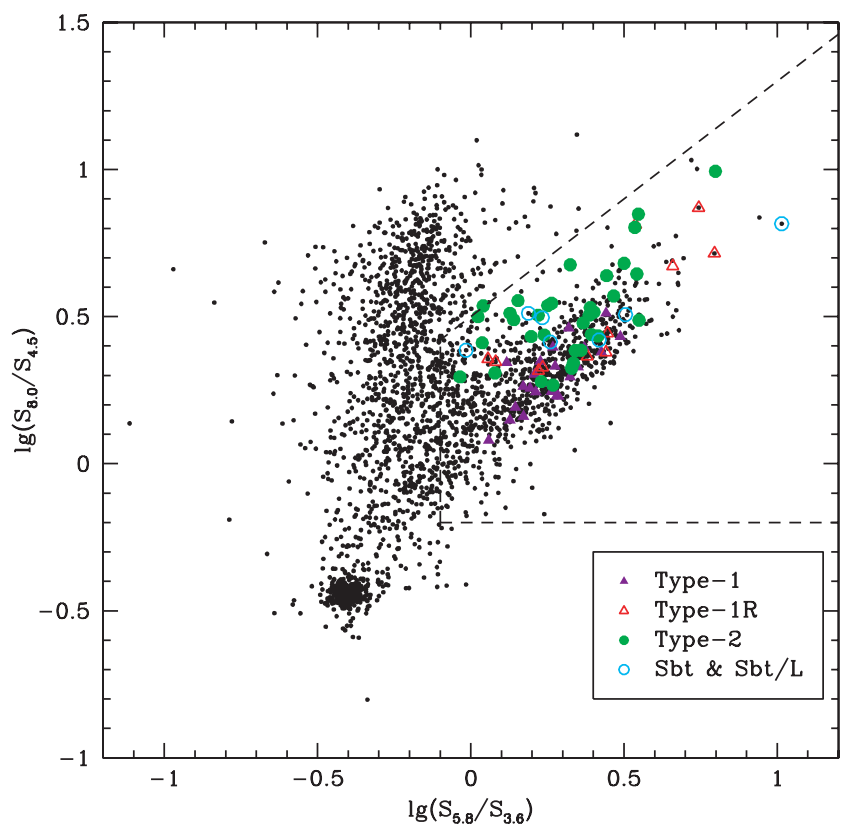

FIG. 1.-IRAC color-color plot for the XFLS field for objects detected in MIPS $24 \mu \mathrm{m}$ and all four IRAC bands. The region within the dashed line is that used to select AGNs. Objects in the AGN samples (both XFLS and SWIRE) are plotted as colored symbols. Object classifications are discussed in $\S 3$.
AGNs with mid-infrared luminosities typical of quasars at moderate redshifts. These objects provide a good complement to the mostly lower luminosity or higher redshift AGNs found in surveys such as the Great Observatories Origins Deep Survey (GOODS) and the Chandra Deep Fields. Preliminary results from the spectroscopic follow-up of the sample presented in Paper I were given in Lacy et al. (2005a). In this paper we describe the selection of samples of mid-infrared-selected AGN candidates flux-limited at $24 \mu \mathrm{m}$ from both the Extragalactic First Look Survey (XFLS) and the Spitzer Wide-area Infrared Extragalactic Survey (SWIRE) $X M M$-Newton Large-Scale Structure Survey (XMM-LSS) fields (Lonsdale et al. 2003) and their spectroscopic follow-up observations. We classify the optical spectra according to the type of AGN and use them to place a lower bound on the fraction of obscured quasars and luminous Seyfert galaxies. We also investigate the X-ray fluxes of those objects that fall on archival observations made with $X M M-N e w t o n$. A more detailed analysis of the spectral energy distributions (SEDs) and X-ray properties of the AGNs will be carried out in a later paper (A. Petric et al. 2007, in preparation). We assume a cosmology with $\Omega_{M}=0.3, \Omega_{\Lambda}=0.7$, and $H_{0}=70 \mathrm{~km} \mathrm{~s}^{-1} \mathrm{Mpc}^{-1}$.

\section{THE SPITZER XFLS AND SWIRE AGN SAMPLES}

We selected samples of AGNs by mid-infrared color from the XFLS and SWIRE XMM-LSS fields. The basis of our colorselection technique is described in Sajina et al. (2005) and Paper I,

TABLE 1

Observing Log for the XFLS AGN Sample

\begin{tabular}{|c|c|c|c|c|c|c|}
\hline Name & Date Observed & Telescope/Instrument ${ }^{\mathrm{a}}$ & Exposure & Slit & Air Mass & P.A. \\
\hline 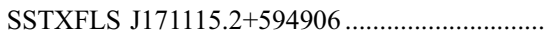 & 2004 Aug 19 & Palomar 200/DbleSpec & $2 \times 600$ & 2.0 & 1.32 & 105 \\
\hline 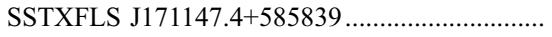 & 2004 Aug 18 & Palomar 200/DbleSpec & $2 \times 600$ & 2.0 & 1.44 & 95 \\
\hline SSTXFLS J171233.4+583610 „........................... & 2005 Oct 30 & Palomar 200/COSMIC & $2 \times 900$ & 2.0 & 1.40 & 90 \\
\hline 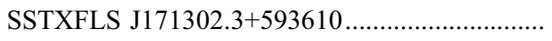 & 2005 Jun 1 & Palomar 200/COSMIC & $2 \times 1200$ & 1.5 & 1.15 & 0 \\
\hline 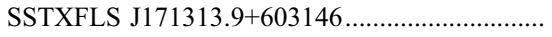 & 2004 Jul 18 & Keck II/ESI & $1 \times 600$ & 1.0 & 1.55 & 127 \\
\hline SSTXFLS J171324.2+585549 „........................... & 2004 Aug 19 & Palomar 200/DbleSpec & $2 \times 600$ & 2.0 & 1.35 & 100 \\
\hline 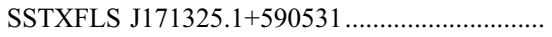 & 2005 Nov 1 & Palomar 200/COSMIC & $1 \times 900$ & 1.5 & 2.14 & 90 \\
\hline 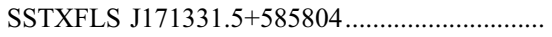 & 2005 Jun 2 & Palomar 200/COSMIC & $2 \times 1200$ & 1.5 & 1.15 & 165 \\
\hline SSTXFLS J171335.1+584756....... & 2005 Jun 1 & Palomar 200/COSMIC & $2 \times 600$ & 1.5 & 1.44 & 90 \\
\hline 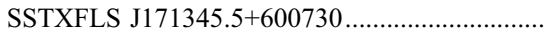 & 2005 Oct 30 & Palomar 200/COSMIC & $2 \times 1800$ & 2.0 & 1.70 & 90 \\
\hline 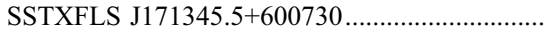 & 2006 Jun 27 & Palomar 200/COSMIC & $3 \times 1800$ & 2.0 & 1.13 & 180 \\
\hline SSTXFLS J171419.9+602724 „......................... & 2005 Jun 2 & Palomar 200/COSMIC & $1 \times 1200$ & 1.5 & 1.18 & 45 \\
\hline 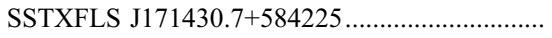 & 2005 Oct 31 & Palomar 200/COSMIC & $2 \times 900$ & 1.5 & 1.86 & 190 \\
\hline 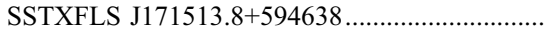 & 2002 Jun 15 & Shane/Kast & $1 \times 1200$ & 1.5 & 1.24 & 73 \\
\hline SSTXFLS J171530.7+600216 .......................... & 2005 Jun 1 & Palomar 200/COSMIC & $1 \times 600$ & 1.5 & 1.15 & 140 \\
\hline 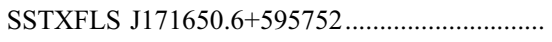 & 2005 Jun 2 & Palomar 200/COSMIC & $1 \times 600$ & 1.5 & 1.23 & 110 \\
\hline 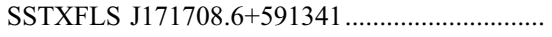 & 2005 Jun 2 & Palomar 200/COSMIC & $1 \times 900$ & 1.5 & 1.26 & 110 \\
\hline SSTXFLS J171750.7+584745 „........................... & 2005 Oct 30 & Palomar 200/COSMIC & $2 \times 1800$ & 2.0 & 1.50 & 90 \\
\hline 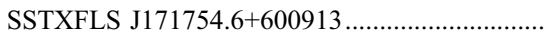 & 2005 Jun 1 & Palomar 200/COSMIC & $2 \times 1200$ & 1.5 & 1.12 & 0 \\
\hline 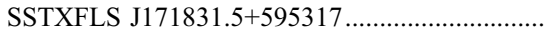 & 2004 Jul 13 & WHT/ISIS & $2 \times 600$ & 2.0 & 1.19 & 5 \\
\hline 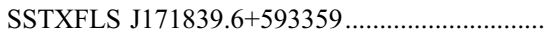 & 2002 Jun 11 & Shane/Kast & $1 \times 1200$ & 1.5 & 1.26 & 109 \\
\hline SSTXFLS J171913.5+584508 …............................ & 2005 Jun 1 & Palomar 200/COSMIC & $2 \times 600$ & 1.5 & 1.18 & 140 \\
\hline SSTXFLS J172044.8+582923 „........................... & 2005 Jun 2 & Palomar 200/COSMIC & $2 \times 1800$ & 2.0 & 1.11 & 90 \\
\hline SSTXFLS J172050.4+591511 _............................. & 2004 Jul 13 & WHT/ISIS & $2 \times 900$ & 2.0 & 1.16 & 85 \\
\hline SSTXFLS J172050.4+591511 „........................... & 2006 Jun 28 & Palomar 200/COSMIC & $3 \times 1800$ & 1.5 & 1.15 & 210 \\
\hline 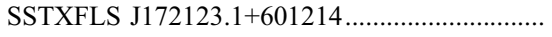 & 2004 Jul 18 & Keck II/ESI & $1 \times 600$ & 1.0 & 1.57 & 125 \\
\hline SSTXFLS J172219.5+594506 _............................ & 2005 Jun 2 & Palomar 200/COSMIC & $2 \times 600$ & 1.5 & 1.48 & 90 \\
\hline 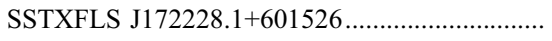 & 2005 Jun 2 & Palomar 200/COSMIC & $1 \times 1200$ & 1.5 & 1.24 & 45 \\
\hline 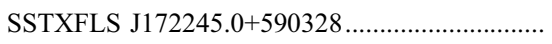 & 2005 Nov 1 & Palomar 200/COSMIC & $2 \times 1200$ & 1.5 & 1.55 & 100 \\
\hline 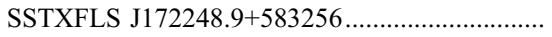 & 2005 Jun 2 & Palomar 200/COSMIC & $2 \times 1200$ & 1.5 & 1.12 & 45 \\
\hline SSTXFLS J172248.9+583256 ........................... & 2006 May 26 & IRTF/SpeX & $22 \times 120$ & 0.5 & 1.48 & 100 \\
\hline SSTXFLS J172328.4+592947 ............................ & 2004 Aug 19 & Palomar 200/DbleSpec & $2 \times 600$ & 2.0 & 1.5 & 90 \\
\hline 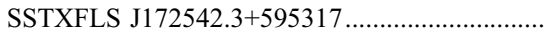 & 2005 Jun 2 & Palomar 200/COSMIC & $2 \times 1200$ & 1.5 & 1.34 & 90 \\
\hline
\end{tabular}

${ }^{\text {a }}$ DbleSpec $=$ Double Spectrograph 
TABLE 2

OBSERVING LOG FOR THE SWIRE $X M M$-LSS SAMPLE

\begin{tabular}{|c|c|c|c|c|c|c|}
\hline Name & Date Observed & Telescope/Instrument & Exposure & Slit & Air Mass & P.A. \\
\hline SWIRE2 J021638.21-042250.8 & 2005 Oct 30 & Palomar 200/COSMIC & $1 \times 900$ & 1.5 & 1.45 & 125 \\
\hline SWIRE2 J021657.77-032459.7 & 2005 Oct 29 & Palomar 200/COSMIC & $1 \times 600$ & 1.5 & 1.84 & 225 \\
\hline SWIRE2 J021729.06-041937.8 & 2005 Oct 30 & Palomar 200/COSMIC & $2 \times 1800$ & 1.5 & 1.4 & 20 \\
\hline SWIRE2 J021749.00-052306.9 & 2005 Nov 1 & Palomar 200/COSMIC & $2 \times 1800$ & 1.5 & 1.30 & 7 \\
\hline SWIRE2 J021759.91-052056.1 & 2005 Oct 31 & Palomar 200/COSMIC & $2 \times 1200$ & 1.5 & 1.28 & 20 \\
\hline SWIRE2 J021809.45-045945.9 & 2005 Oct 31 & Palomar 200/COSMIC & $2 \times 1200$ & 1.5 & 1.31 & 20 \\
\hline SWIRE2 J021859.74-040237.2 & 2005 Oct 30 & Palomar 200/COSMIC & $1 \times 600$ & 1.5 & 1.89 & 312 \\
\hline SWIRE2 J021909.60-052512.9 & 2005 Oct 29 & Palomar 200/COSMIC & $1 \times 600$ & 1.5 & 1.31 & 150 \\
\hline SWIRE2 J021938.70-032508.2 & 2005 Nov 1 & Palomar 200/COSMIC & $1 \times 600$ & 1.5 & 1.40 & 42 \\
\hline SWIRE2 J021939.08-051133.8 & 2005 Oct 29 & Palomar 200/COSMIC & $1 \times 600$ & 1.5 & 1.36 & 90 \\
\hline SWIRE2 J022005.93-031545.7 & 2005 Nov 1 & Palomar 200/COSMIC & $1 \times 600$ & 1.5 & 1.55 & 47 \\
\hline SWIRE2 J022012.21-034111.8 & 2005 Nov 1 & Palomar 200/COSMIC & $1 \times 600$ & 1.5 & 1.74 & 47 \\
\hline SWIRE2 J022039.48-030820.3 & 2005 Oct 31 & Palomar 200/COSMIC & $1 \times 900$ & 1.5 & 1.50 & 140 \\
\hline SWIRE2 J022133.82-054842.8 & 2005 Nov 1 & Palomar 200/COSMIC & $1 \times 600$ & 1.5 & 1.75 & 145 \\
\hline SWIRE2 J022211.57-051308.1 & 2005 Nov 1 & Palomar 200/COSMIC & $2 \times 1800$ & 1.5 & 1.31 & 341 \\
\hline SWIRE2 J022225.86-050015.1 & 2005 Oct 31 & Palomar 200/COSMIC & $1 \times 600$ & 1.5 & 2.24 & 48 \\
\hline SWIRE2 J022255.87-051351.7 & 2005 Oct 31 & Palomar 200/COSMIC & $1 \times 900$ & 1.5 & 1.49 & 30 \\
\hline SWIRE2 J022301.97-052335.8 & 2005 Nov 1 & Palomar 200/COSMIC & $2 \times 1200$ & 1.5 & 1.46 & 341 \\
\hline SWIRE2 J022306.74-050529.1 & 2005 Oct 30 & Palomar 200/COSMIC & $2 \times 900$ & 1.5 & 1.76 & 315 \\
\hline SWIRE2 J022310.42-055102.7 & 2005 Oct 29 & Palomar 200/COSMIC & $2 \times 1800$ & 2.0 & 1.24 & 90 \\
\hline SWIRE2 J022348.96-025426.0 & 2005 Nov 1 & Palomar 200/COSMIC & $1 \times 900$ & 1.5 & 1.47 & 145 \\
\hline SWIRE2 J022356.49-025431.1 & 2005 Oct 29 & Palomar 200/COSMIC & $2 \times 1800$ & 2.0 & 1.24 & 90 \\
\hline SWIRE2 J022422.27-031054.7 & 2005 Oct 31 & Palomar 200/COSMIC & $1 \times 600$ & 1.5 & 1.67 & 48 \\
\hline SWIRE2 J022431.58-052818.8 & 2005 Oct 31 & Palomar 200/COSMIC & $1 \times 600$ & 1.5 & 1.7 & 310 \\
\hline SWIRE2 J022438.97-042706.3 & 2005 Nov 1 & Palomar 200/COSMIC & $1 \times 600$ & 1.5 & 1.95 & 45 \\
\hline SWIRE2 J022455.77-031153.6 & 2005 Oct 31 & Palomar 200/COSMIC & $1 \times 900$ & 1.5 & 1.52 & 40 \\
\hline SWIRE2 J022508.33-053917.7 & 2005 Oct 31 & Palomar 200/COSMIC & $1 \times 900$ & 1.5 & 1.32 & 26 \\
\hline SWIRE2 J022542.02-042441.1 & 2005 Oct 30 & Palomar 200/COSMIC & $2 \times 900$ & 1.5 & 1.6 & 40 \\
\hline SWIRE2 J022600.01-035954.5 & 2005 Oct 31 & Palomar 200/COSMIC & $1 \times 900$ & 1.5 & 1.45 & 125 \\
\hline SWIRE2 J022612.67-040319.4 & 2005 Oct 31 & Palomar 200/COSMIC & $1 \times 600$ & 1.5 & 1.88 & 48 \\
\hline SWIRE2 J022700.77-042020.6 & 2005 Oct 29 & Palomar 200/COSMIC & $1 \times 600$ & 1.5 & 1.32 & 150 \\
\hline
\end{tabular}

although the samples in this paper are flux-limited at $24 \mu \mathrm{m}$ rather than at $8 \mu \mathrm{m}$, as was the case for the sample of Paper I. To obtain the XFLS sample, we began by matching the XFLS $24 \mu \mathrm{m}$ catalog of Fadda et al. (2006) to the Infrared Array Camera (IRAC) four-band catalog of Lacy et al. (2005b). All MIPS $24 \mu \mathrm{m}$ detections with detections in all four IRAC bands were then plotted on the color-color diagram of Figure 1. For the SWIRE XMMLSS sample, we used the band-merged SWIRE IRAC-MIPS $24 \mu \mathrm{m}$ catalog available from the Spitzer popular products Web site. ${ }^{4}$ The color cut used to select AGN candidates was $\log \left(S_{5.8} / S_{3.6}\right)>$ $-0.1, \log \left(S_{8.0} / S_{4.5}\right)>-0.2$, and $\log \left(S_{8.0} / S_{4.5}\right) \leq 0.8 \log \left(S_{5.8} /\right.$ $\left.S_{3.6}\right)+0.5$. The $\log \left(S_{5.8} / S_{3.6}\right)$ cut was moved by +0.1 compared to Paper I. The exact position of this cut is not critical, and it was felt that shifting this would help remove non-AGN contaminants, particularly as the final $5.8 \mu \mathrm{m}$ fluxes in the XFLS catalog were increased by $\approx 10 \%$ in the final version of the XFLS catalog relative to the catalog used in Paper I. We discuss the exact placement of the color cuts with respect to the completeness of the AGN selection in $\S 5.3$. Candidates were selected down to limiting $24 \mu \mathrm{m}$ fluxes of $4.4 \mathrm{mJy}$ in the XFLS and $6.6 \mathrm{mJy}$ in the SWIRE $X M M$-LSS fields.

The $24 \mu \mathrm{m}$ flux-limited sample should be an improvement on $8 \mu \mathrm{m}$ selection by making us more sensitive to highly reddened objects and high-redshift objects, where the strong $k$-correction on the mid-infrared dust emission means that they drop out of flux-limited samples selected at shorter wavelengths. The disadvantage of this selection is that we will miss objects at $z \sim 1.5$

\footnotetext{
${ }^{4}$ See http://data.spitzer.caltech.edu/popular/.
}

that have strong silicate absorption in the $24 \mu \mathrm{m}$ band; however, as our median redshift is $\approx 0.6$, only a few objects will be missed due to this effect. The XFLS sample is complete for objects falling in the IRAC "AGN wedge" color selection to a flux limit of $4.6 \mathrm{mJy}$ at $24 \mu \mathrm{m}$, and the SWIRE sample is complete to a flux limit of $6.6 \mathrm{mJy}$ (note that all MIPS sources down to these flux limits have four-band IRAC detections). Approximately $20 \%$ of the MIPS $24 \mu \mathrm{m}$ sources at these flux levels fall into our AGNdominated part of the color-color plot.

A few stars and nearby galaxies have been removed from the samples. Most stars were only present in the initial selection due to photometric errors related to saturation in the shorter IRAC bands, although one star (SSTXFLS J172432.8+592646; see Paper I) has the correct colors to fall in the sample, presumably due to a dust disk. Very low redshift $(z \lesssim 0.05)$ galaxies are also possible contaminants due to the presence of $6.2 \mu \mathrm{m}$ polycyclic aromatic hydrocarbon (PAH) emission in the $5.8 \mu \mathrm{m}$ band of IRAC (Sajina et al. 2005). The interacting galaxy Arp 54 appeared in the selection of the SWIRE AGN sample but has been removed for this reason.

Tables 1 and 2 contain the observing logs for our samples. Most data were taken with the COSMIC instrument on the Palomar 200 inch $(5 \mathrm{~m})$ telescope. Some spectra were obtained with the ISIS instrument at the $4.2 \mathrm{~m}$ William Herschel Telescope (WHT) on La Palma, with the Echellette Spectrograph and Imager (ESI) at the Keck II telescope, or with the Kast spectrograph at the $3 \mathrm{~m}$ Shane Telescope at Lick Observatory. SSTXFLS J172248.9+ 583256 , for which we failed to obtain a redshift in the optical, was observed in the near-infrared using the low-dispersion mode of 

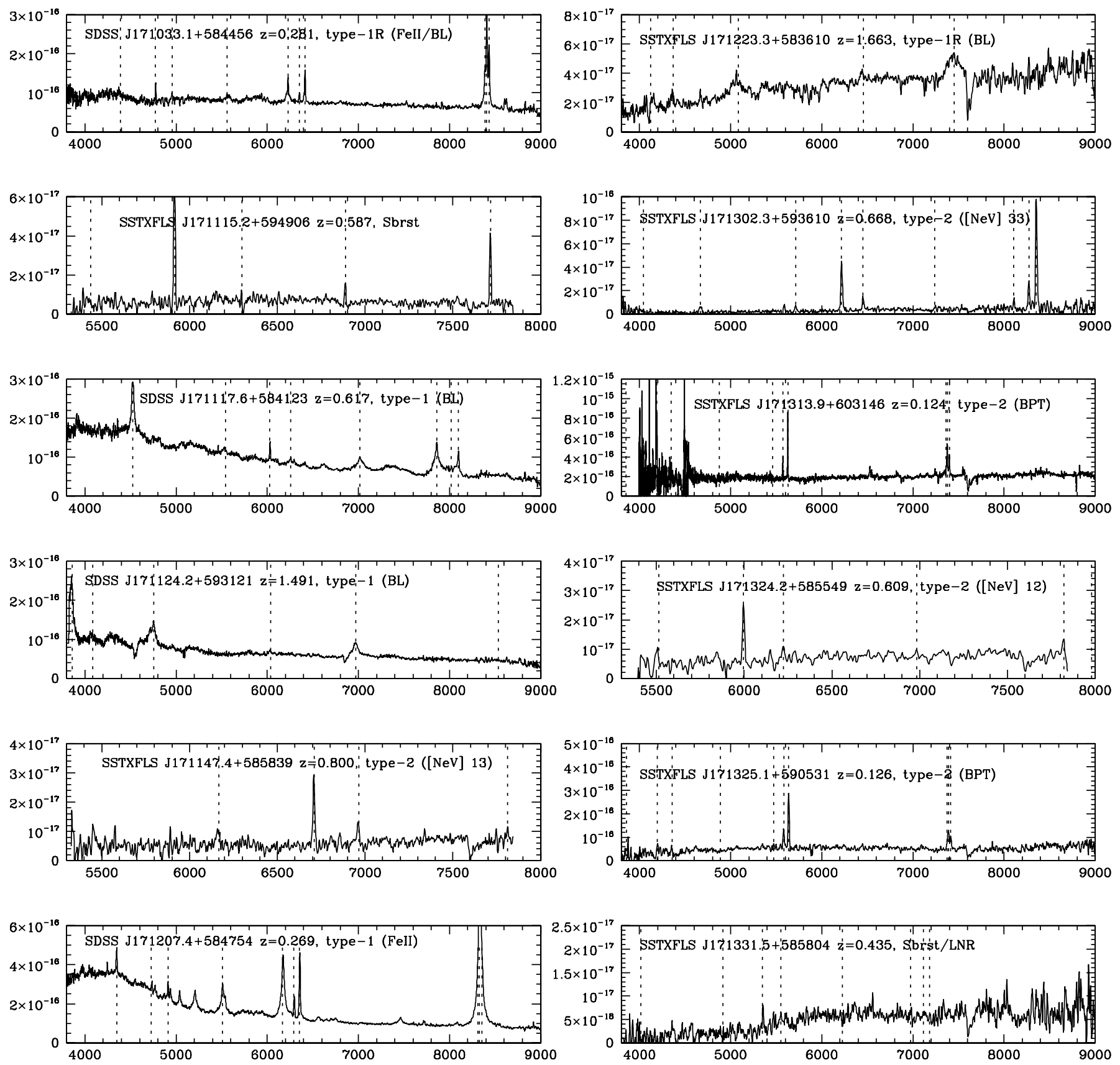

FIG. 2.-Optical spectra of the XFLS AGN candidates. Dotted lines indicate the positions of redshifted emission lines (not all of which are necessarily detected in a given spectrum). The emission lines indicated are (from blue to red): Ly $\alpha, \mathrm{N}$ v $\lambda 1240, \mathrm{C}$ IV $\lambda 1549, \mathrm{He}$ II $\lambda 1640, \mathrm{C}$ III] $\lambda 1909$, [ $\mathrm{Ne}$ IV] $\lambda 2424, \mathrm{Mg}$ II $\lambda 2798$, [Ne v]

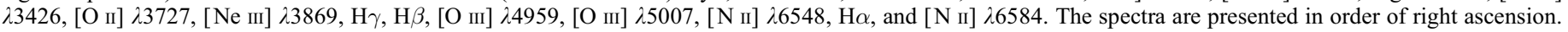

the SpeX instrument at the Infrared Telescope Facility (IRTF; Rayner et al. 2003). Data were analyzed in IRAF using standard routines for flat-fielding, wavelength calibration, and sky subtraction. No attempt has been made to correct for reddening. An extinction correction has been applied based on air mass, but no attempt has been made to correct the telluric absorption features. The spectra are approximately photometrically calibrated, but variable seeing and cirrus clouds means that we expect our spectrophotometric accuracy to be no better than 50\%. In the XFLS field most quasars have spectra from the SDSS, which have been used where they exist. A few of the quasars in the SWIRE field have redshifts from the literature. Several of our XFLS sources have spectra in the MMT spectroscopic survey of Papovich et al. (2006), including one object missed from our original selection that is within the flux-limited sample (SSTXFLS J171454.4+ 584948). The spectra of all the objects, with the exception of the SWIRE quasars with redshifts from the literature, are shown in Figures 2 and 3. Objects with spectral classifications from both this paper and Papovich et al. (2006) are discussed in Appendix A. For completeness, Appendix B contains details of the results of spectroscopy on the objects in the $8 \mu \mathrm{m}$ sample of Paper I that are too faint at $24 \mu \mathrm{m}$ to fall into the XFLS sample.

\section{RESULTS}

\subsection{Optical Spectral Classification of the AGN Candidates}

We have used a combination of standard classification schemes to classify our AGN candidates, principally because the large 

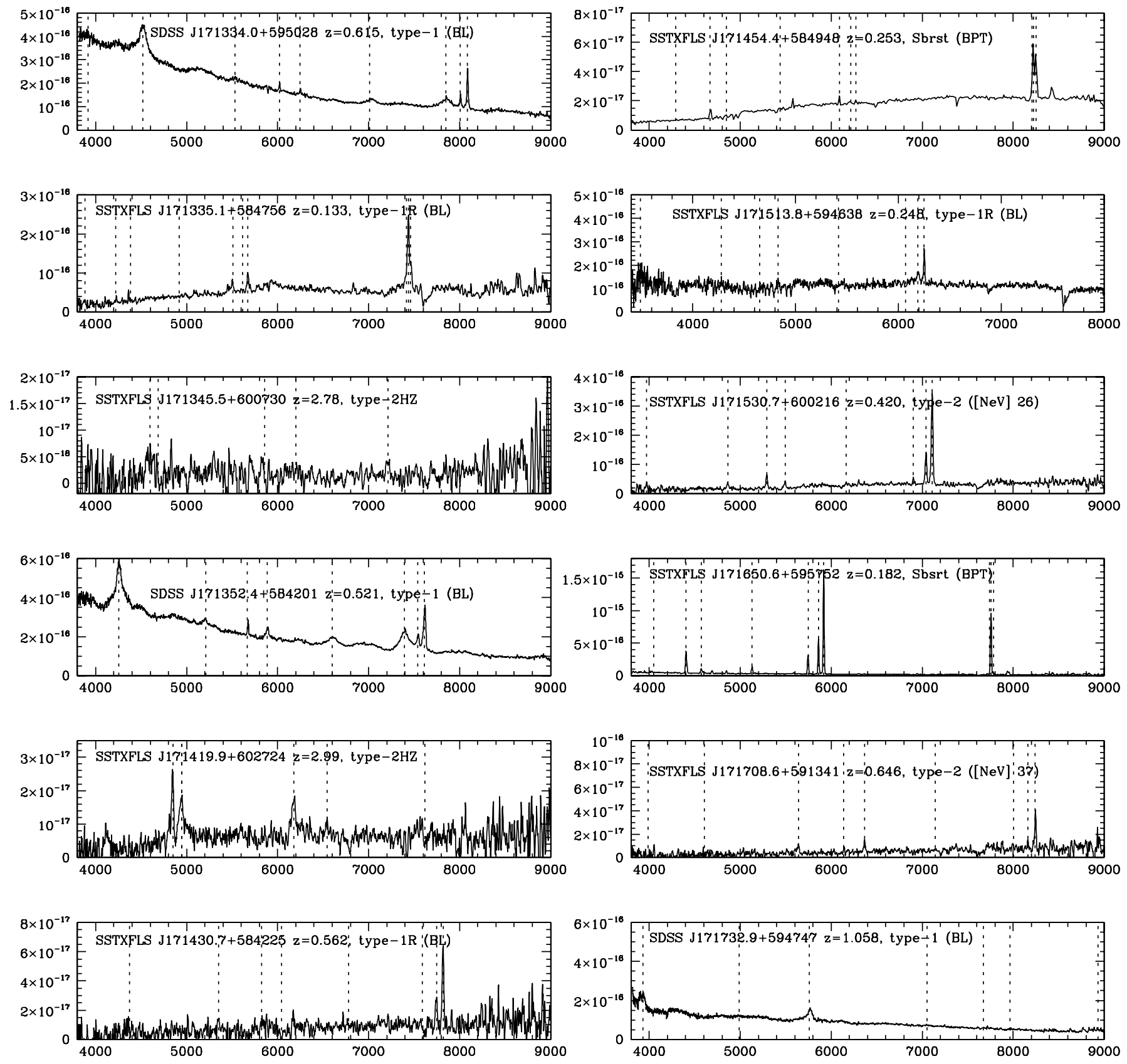

Fig. 2.-Continued

redshift and luminosity range of our sample makes any single scheme unworkable. We divide our objects into four main classes. Normal quasars and Seyfert 1 galaxies are assigned type 1. The obscured quasars are subdivided into three classes. Reddened quasars, showing broad lines in their optical spectra but with a faint, presumably dust-reddened quasar continuum and/or clear host galaxy emission with no sign of a blue quasar continuum above the host galaxy light, are classified as type 1R. Objects with highionization narrow emission lines that cannot be produced in a pure starburst scenario are classified as type 2. Objects with starburst emission-line ratios in emission-line diagnostic diagrams (Baldwin et al. 1981, hereafter BPT) and/or low-ionization spectra with strong Balmer lines are classified as starbursts (Sbt). Where we have a low-ionization spectrum with too few lines to plot on a diagnostic diagram, or the lines are low-ionization and have $\mathrm{H} \alpha$ beyond the range of the optical spectrum, they are clas- sified as starburst/LINER (Sbt/L). Details of the bases of our optical classifications are described below. It should be emphasized that these classes are based on the observed-frame optical spectra; the rest-frame optical spectra may give different classifications. The classifications and their bases are given in the final columns of Tables 3 and 4 . Of our sample of 77 objects, we find 25 objects with type 1 optical spectra, 11 with type 1R, 34 with type 2, 2 with unambiguous starburst spectra, and 5 with spectra that could be from either starbursts or LINERs.

\subsection{Type 1 Objects}

Objects with line widths of at least one permitted emission line $>3000 \mathrm{~km} \mathrm{~s}^{-1}$ are classified as type 1 . These objects have "BL" in the classification basis column in Tables 3 and 4 . This is sufficient to distinguish most of the luminous quasars. However, as our sample spans a wide luminosity range, several of the lower 

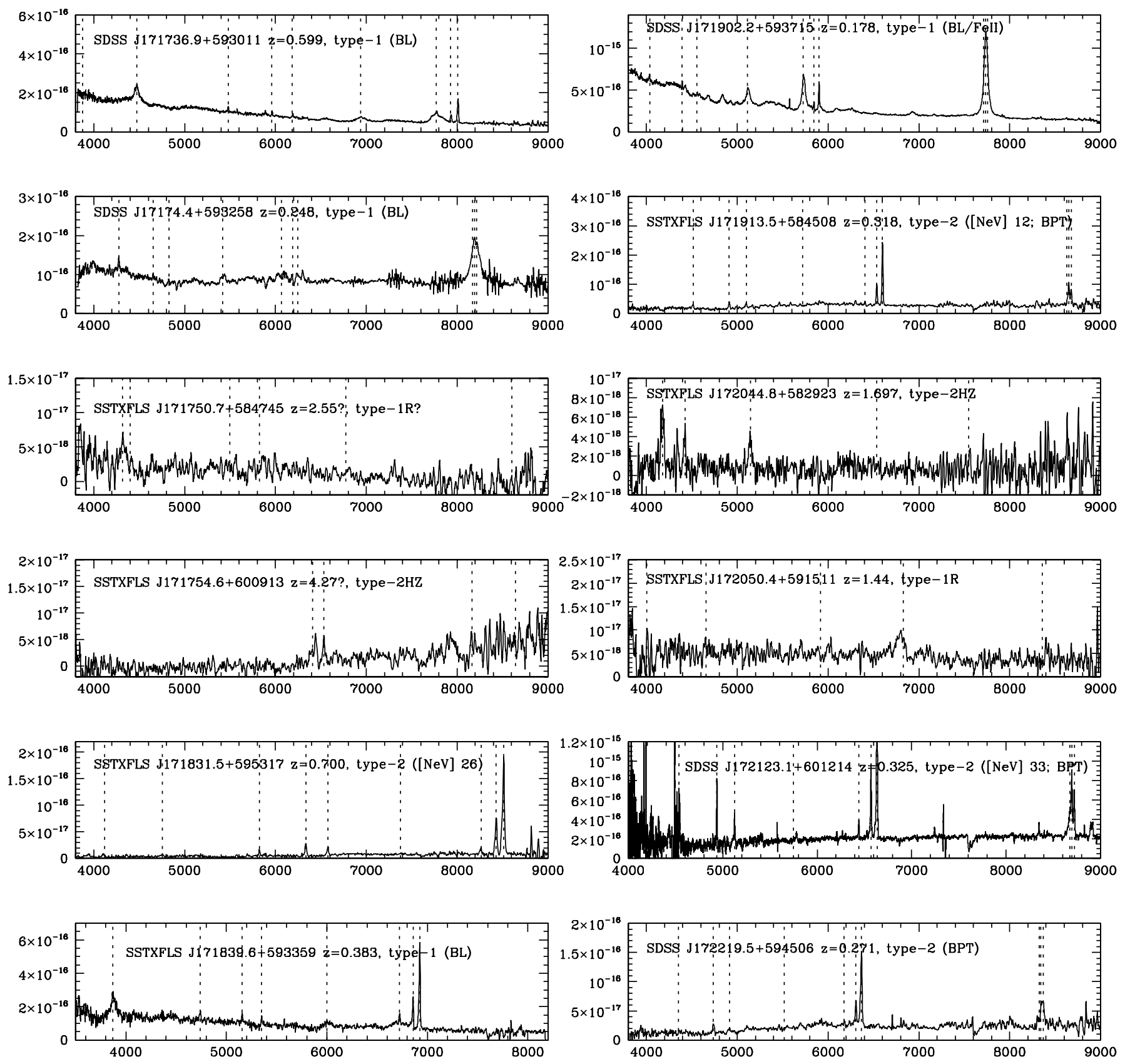

FIG. 2.-Continued

luminosity, lower redshift type 1 objects have narrower permitted lines (including a couple of candidate narrow-line Seyfert 1 galaxies). We therefore use the presence of strong permitted lines $(\mathrm{H} \beta /[\mathrm{O}$ III $] \lambda 5007>0.5)$ and Fe II emission in the 4000-5500 $\AA$ range to classify them as type 1 . These objects have "Fe II" in the basis column. The division into normal, type 1, or reddened, type $1 \mathrm{R}$, quasars is also made. This is decided based on one of two criteria. If the optical spectrum is quasar-like, with featureless continuum and broad emission lines, but the continuum appears significantly reddened $[E(B-V) \gtrsim 0.3]$ compared to that of a normal quasar (see $\S 3.5$ ), then the object is classified as type 1R. Also, some low-z objects were classified as type $1 \mathrm{R}$ if the optical spectrum contains broad lines, but host galaxy features are visible and the object is dominated by host galaxy light in the blue.

\subsection{Type 2 Objects}

Objects without broad lines or Fe II emission but with highionization narrow lines are classified as follows. Objects with [Ne v] detections with rest-frame equivalent width $>5 \AA$ are classified as type 2 , with the basis column containing " $[\mathrm{Ne} \mathrm{v}]$ " and the measured rest-frame equivalent width in angstroms (for comparison, the [ $\mathrm{Ne} \mathrm{v}] \lambda 3426$ emission line in the SDSS composite quasar spectrum of Vanden Berk et al. [2001] has an equivalent width of $\approx 0.6 \AA$ ). For low-redshift objects in which $\mathrm{H} \alpha$ was included in the spectra, we have used BPT diagrams to classify the AGNs. Figure 4 shows the emission-line ratios of our objects plotted on two diagnostic diagrams using the divisions between star-forming, Seyfert, and LINER galaxies proposed by Kewley et al. (2006). Most objects plot with the Seyfert galaxies, 

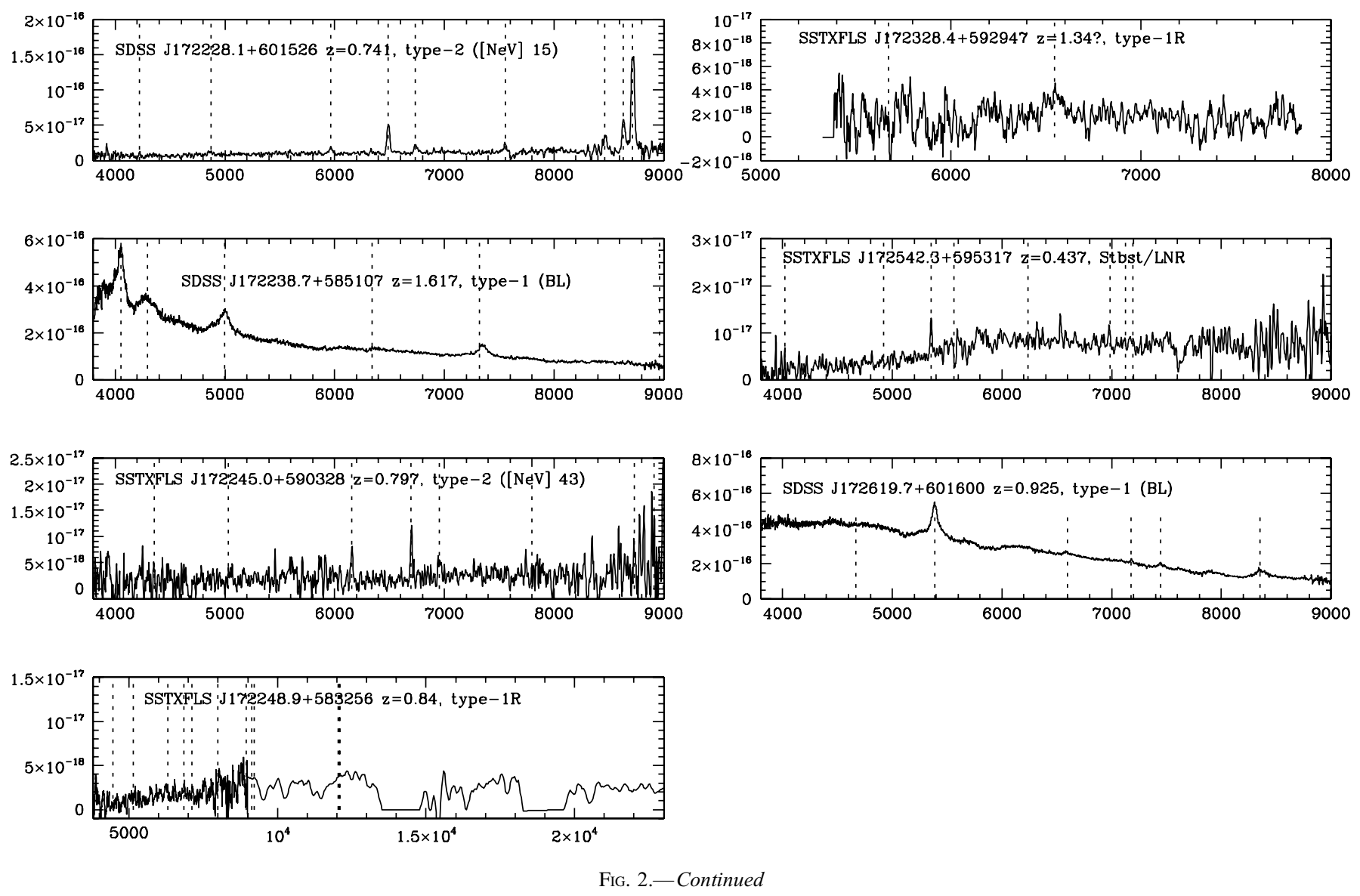

although two objects plot close to the AGN-starburst division line in both plots and are presumably composite starburst-AGN objects (SSTXFLS 171650.6+595752 and SWIRE2 J022225.86$050015.1)$. We classify these as type 2 , as they do have evidence of AGN activity, even if this is not dominating their optical emission-line ratios. For some objects with neither [ $\mathrm{Ne} v$ ] nor $\mathrm{H} \alpha /[\mathrm{N}$ II $]$ detected in their spectra, we have relied on the ionization parameter as an approximate AGN indicator and used the [O III] $\lambda 5007 /[\mathrm{O}$ II $] \lambda 3727$ ratio, classifying objects with [O III] $\lambda 5007 /\left[\mathrm{O}_{\mathrm{II}}\right] \lambda 3727>1$ as questionable type 2 objects.

There are also a few high-redshift objects having high-ionization UV permitted lines with widths of $\sim 2000 \mathrm{~km} \mathrm{~s}^{-1}$. Line widths of this order are seen in the UV spectrum of NGC 1068 (Grimes et al. 1999). Given this and their high luminosities, we anticipate that the true broad-line widths will be much higher, so we are probably seeing only the narrow-line region in our spectra. However, given their high redshifts $(z>1.5)$, only a very small amount of extinction is required to hide the broad-line region in the restframe UV. For the three highest redshift objects, at 2.78, 2.99, and 4.27 , the rest-frame optical to near-infrared light is sampled in the IRAC bands, and this must almost certainly be dominated by reddened quasar light for these objects to exceed our IRAC flux limits. It is therefore unclear whether these objects should be classified as type 2 (showing no strong broad emission lines or quasar continuum in the rest-frame optical) or type 1R. We thus assign $z>1.5$ objects with narrow UV lines to type $2 \mathrm{HZ}$ (and group them with the type 2 objects for plotting and statistical purposes), pending better information on their rest-frame optical emission-line properties and SEDs from near-infrared imaging and spectroscopy. In all of these objects, $\operatorname{Ly} \alpha$ is typically faint compared to the high- ionization UV lines, probably due to absorption by neutral hydrogen (e.g., van Ojik et al. 1994).

\subsection{Starbursts and LINERs}

We are left with seven objects with low-ionization lines showing no signs of obvious AGN activity. Such objects have been classified as type 3 AGNs by Leipski et al. (2005). They are all relatively low-redshift $(z<0.6)$. All show [O II] $\lambda 3727$ in emission and Balmer absorption features. One also shows strong narrow $\mathrm{H} \beta$ and $\mathrm{H} \gamma$ emission. The two objects we have been able to positively identify as starbursts in the optical have been classified as such. The remainder are classified as starbursts/LINERs. Note that we fail to find any unambiguous LINERs in our sample. (SSTXFLS 171454.4+584948 plots below the Seyfert galaxies in the $[\mathrm{N}$ II $] / \mathrm{H} \alpha$ vs. $\left[\mathrm{O}_{\mathrm{III}}\right] / \mathrm{H} \beta$ diagram, in the traditional LINER region [e.g., Kauffmann et al. 2003] but well within the starbursts in the $\left[\mathrm{O}_{\mathrm{I}}\right] / \mathrm{H} \alpha$ vs. $[\mathrm{O}$ III $] / \mathrm{H} \beta$ diagram, which gives a better discrimination of LINERs according to Kewley et al. [2006], so we have classified it as a starburst.) This may be due to LINERs lacking a substantial hot torus bright enough to dominate the midinfrared over stellar emission (e.g., Ogle et al. 2006), but better spectra of our starburst/LINER objects are needed to confirm this.

For the most part our classifications are consistent with those of Papovich et al. (2006), although there are some objects that are classified differently. These are discussed in Appendix A.

\subsection{Optical to Mid-Infrared Color}

In Figure 5 we show the observed $R-[8.0]$ color plotted against redshift for the different spectral classifications. For the 

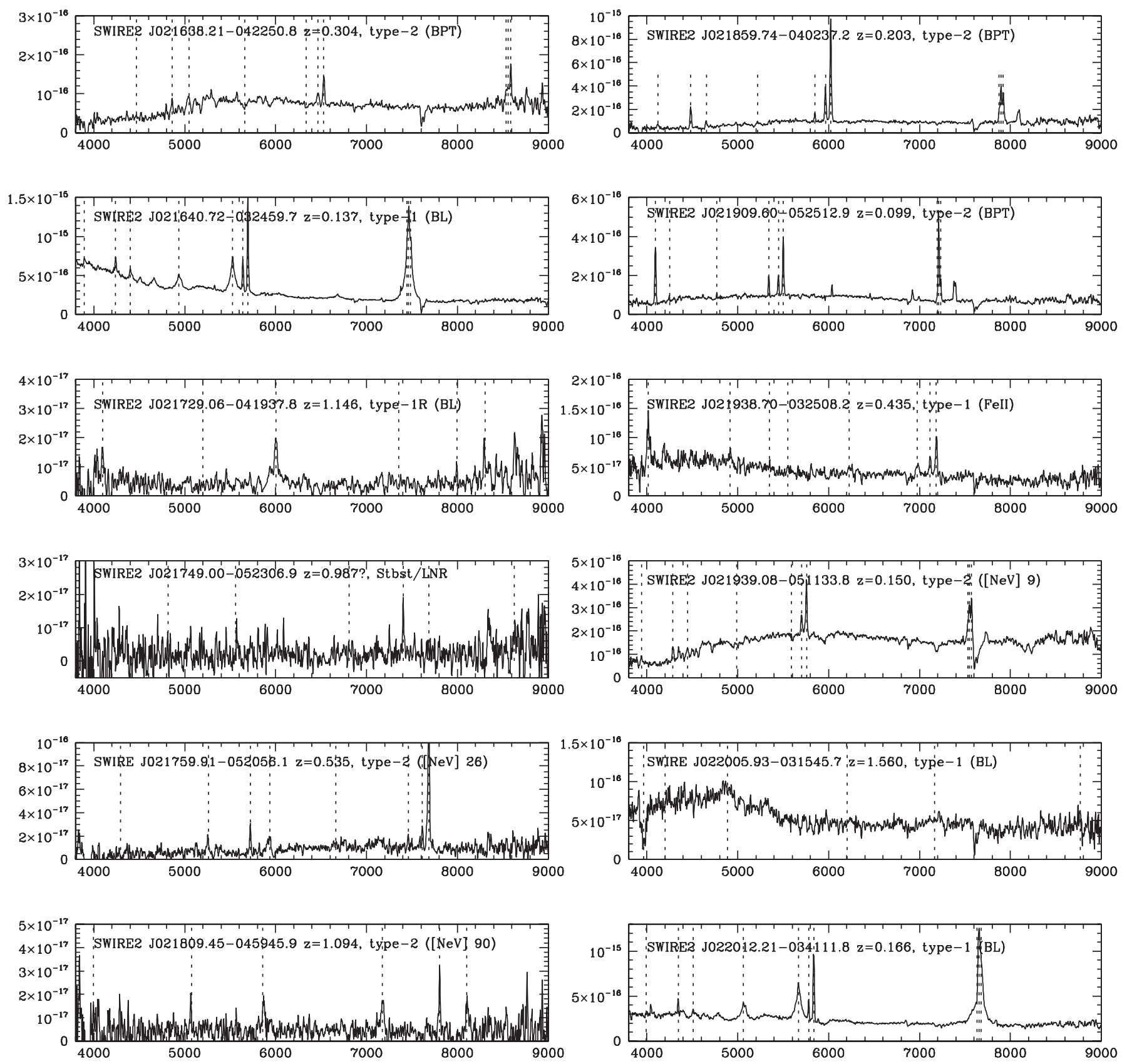

FIG. 3.-Optical spectra of the SWIRE AGN candidates. The positions of emission lines are indicated as in Fig. 2. The spectra are presented in order of right ascension.

XFLS sample the $R$ magnitudes are from the survey of Fadda et al. (2004); those for the SWIRE sample are from the Cambridge automatic plate measuring scans of the UK Schmidt telescope plates (although in a few cases of faint or blended sources the magnitudes have been estimated by eye from digitized sky survey plates). Apart from a couple of low-redshift AGNs, which probably have their optical emission dominated by their host galaxies, all the normal type 1 objects trace the expected color locus for type 1 quasars very well, particularly the "IR-luminous" SED of Richards et al. (2006), suggesting that the mid-infrared selection is not picking a very unusual population of type 1 quasars but rather is finding quasars with the properties that we would expect from a sample selected in the mid-infrared. After the host galaxy contribution to the $R$ band becomes negligible at $z \gtrsim 0.4$, the nor- mal and obscured (types 1R, 2, and 3) quasars separate, with very few objects having moderately red colors. This relatively clean separation shows that our optical spectral classifications are indeed likely to be correct.

The $z>1.5$ type 2 objects (type $2 \mathrm{HZ}$ in Table 3 ), which have intermediate-UV line widths and whose precise optical classification is therefore unclear, are obviously redder than the expected mid-infrared-to-optical color of normal quasars at these redshifts, suggesting that they are not simply quasars with unusually narrow lines but do indeed have most of their nuclear emission extinguished by dust.

Our division between type 1 and type 1R quasars also appears to be justified on the basis of this color (at least for objects at $z \gtrsim 0.4$, where the host galaxy does not affect the $R$ magnitude), 



FIG. 3.-Continued

with the type $1 \mathrm{R}$ quasars having rest-frame reddenings $E(B-$ $V) \sim 0.3-0.7$.

\section{X-RAY DETECTIONS}

There is some overlap between objects in the SWIRE XMMLSS fields and archival XMM-Newton data. Twenty-one of 34 sources in the SWIRE $X M M$-LSS sample fall within moderately deep (5-50 ks) XMM-Newton exposures. These are sufficiently deep to detect or set useful limits on the X-ray flux from our sources. These data appear to have been taken for several different surveys, including the $X M M$-LSS (e.g., Chiappetti et al. 2005) and the Subaru XMM-Newton Deep Survey (Watson et al. 2004). We have obtained approximate fluxes for these objects in order to investigate the optical-to-X-ray luminosity ratios of our sources (Table 5). This allows us to place our mid-infrared-selected obscured AGNs into the context of X-ray samples of similar objects. Counts in the $0.2-12 \mathrm{keV}$ range from the pn detector of the European Photon Imaging Camera, as listed in the 1XMM serendipitous catalog (e.g., Michel et al. 2004) or the prerelease of the 2XMM catalog (Second XMM-Newton Serendipitous Source Prerelease Catalogue [2006, XMM-Newton Survey Science Centre]), have been used where available; otherwise, counts have been taken from the source lists available from the $X M M$ Newton archive (the pipeline products [PPS] lists). Limits are estimated from the fluxes of the faintest believable detections on each image and correspond to $\approx 3 \sigma$. Fluxes from the $1 \mathrm{XMM}$ or $2 \mathrm{XMM}$ catalogs are from the pn count rate in $1 \mathrm{XMM}$ and from the average over all detectors in 2XMM. Where only counts or a limit are available, fluxes are derived from the count rate using the PIMMS Web site, ${ }^{5}$ assuming an input spectrum with a photon index of 2 and a column of $10^{21} \mathrm{~cm}^{-2}$.

\footnotetext{
${ }^{5}$ See http://heasarc.gsfc.nasa.gov/Tools/w3pimms.html.
} 

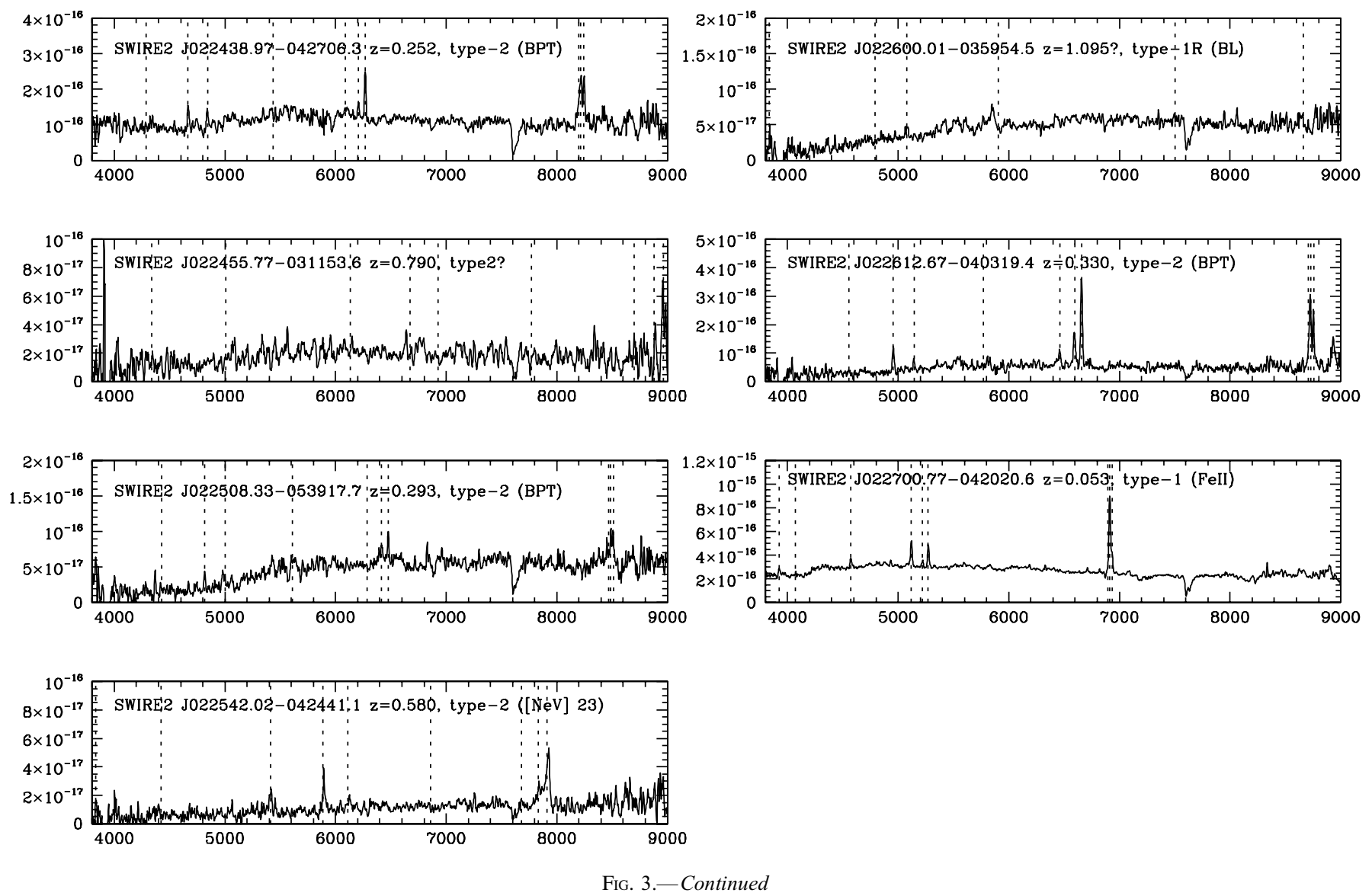

A few points are clear from even this preliminary study. All the type 1 quasars are detected in the X-ray (albeit with about an order of magnitude of scatter in their X-ray-to-infrared ratios), but only about half of the optically obscured quasars (Fig. 6) are detected. The obscured quasars do, however, show a wide range in X-ray-to-infrared flux ratios, ranging from little or no absorption to X-ray fluxes absorbed by a factor of 10 or more (averaged over the $0.2-12 \mathrm{keV}$ band) relative to the type 1 objects. Interestingly, one of the two starburst/LINER objects in these X-ray fields is detected, although the other is not.

\section{DISCUSSION}

\subsection{Selection Effects and Redshift Biases}

Every technique for selecting AGNs is affected by selection biases, and this one is no exception. The color selection means that objects whose observed mid-infrared colors are not dominated by thermal emission from the AGN will be missing from the sample. This can happen in a variety of ways. First, the AGN may simply be of such low luminosity in the infrared that the starlight from the host galaxy dominates the mid-infrared emission, either because the AGN is intrinsically weak or because the covering factor of the hot dust is low, perhaps because of an unusual torus geometry. This bias gets worse with increasing redshift as the stellar bump in the rest-frame near-infrared is redshifted into the mid-infrared bands, and the $k$-correction on the thermal emission of the dust becomes large. Second, some AGNs will simply be so highly obscured that the AGN emission is not seen at observed wavelengths $<8 \mu \mathrm{m}$. Third, some AGNs may also be so highly obscured that they drop out of the IRAC catalogs at short wavelengths.
The positions of the different classes of objects within the AGN wedge on the IRAC color-color plot of Figure 1, shown in detail in Figure 7, can give us some idea of the particular selection effects for each class of object. The type 1 quasars all lie in a fairly tight group in the center of the wedge, so few are likely to be missing (see also Paper I). Reddened type 1 objects show more dispersion, including two with very red IRAC colors. The type 2 objects are also more dispersed, with several (mostly low-redshift objects) trending toward the diagonal divide in the color-color plot, presumably due to PAH emission, and several with very red colors. The starbursts and starbursts/LINERs are surprisingly close to the center of the wedge, although one does have extremely red IRAC colors. From Figure 7 we can deduce that some (probably mostly lower $z$ ) type 2 objects are probably missing due to plotting with the PAH-dominated galaxies, and that all classes of obscured quasars are probably missing their reddest representatives. There is a weak trend whereby the higher redshift objects tend to be located toward redder colors in the wedge, although at the highest redshifts, where the IRAC colors are sampling the rest-frame optical and near-infrared, the colors become bluer again.

As these selection effects have yet to be modeled in depth, we will defer further discussion to a later paper, in which we will attempt to model the emission from our objects in detail and apportion luminosity between AGN and starburst components (A. Petric et al. 2007, in preparation). However, we point out here that our fraction of obscured quasars is necessarily a lower limit, and this limit becomes increasingly poor with increasing redshift.

\subsection{Objects with Low-Ionization Optical Spectra}

Are there any high-luminosity AGNs in which both the broadand narrow-line regions are obscured or otherwise missing? This 
TABLE 3

BRIGHT AGNs IN the XFLS REGION

\begin{tabular}{|c|c|c|c|c|}
\hline Name & $\begin{array}{c}S_{24.0} \\
(\mathrm{mJy})\end{array}$ & Redshift & Class & Basis \\
\hline SDSS J171033.1+584456 & 6.1 & 0.281 & $1 \mathrm{R}$ & $\mathrm{Fe}$ II $/ \mathrm{BL}$ \\
\hline 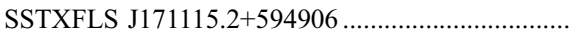 & 9.4 & 0.587 & Sbt & Strong Balmer lines \\
\hline 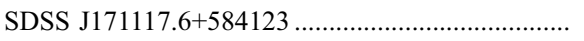 & 5.8 & 0.617 & 1 & $\mathrm{BL}$ \\
\hline 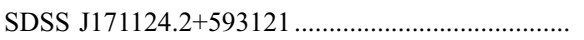 & 5.5 & 1.491 & 1 & BL (LoBAL? $)^{\mathrm{a}}$ \\
\hline 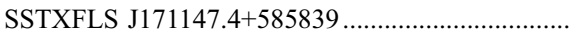 & 4.8 & 0.800 & 2 & {$[\mathrm{Ne} \mathrm{v}] 13$} \\
\hline SDSS J171207.4+584754 & 13.4 & 0.269 & 1 & $\mathrm{Fe}$ II \\
\hline 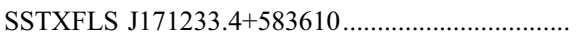 & 5.1 & 1.663 & $1 \mathrm{R}$ & BL \\
\hline SSTXFLS J171302.3+593610 & 11.8 & 0.668 & 2 & {$[\mathrm{Ne} v] 33$} \\
\hline 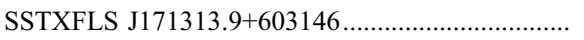 & 10.5 & 0.124 & 2 & BPT \\
\hline 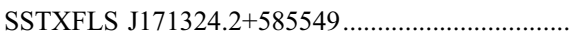 & 4.9 & 0.609 & 2 & {$[\mathrm{Ne} \mathrm{v}] 12$} \\
\hline 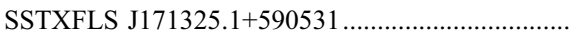 & 9.5 & 0.126 & 2 & $\mathrm{BPT}$ \\
\hline 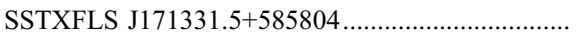 & 5.9 & 0.435 & $\mathrm{Sbt} / \mathrm{L}$ & {$[\mathrm{O}$ III $] /[\mathrm{O}$ II $]<1$} \\
\hline SDSS J171334.0+595028 & 5.4 & 0.615 & 1 & $\mathrm{BL}$ \\
\hline 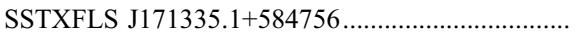 & 23.7 & 0.133 & $1 \mathrm{R}$ & $\mathrm{Fe}$ II \\
\hline 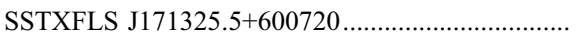 & 4.9 & 2.78 & $2 \mathrm{HZ}$ & Narrow UV lines \\
\hline 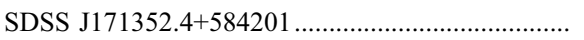 & 23.9 & 0.521 & 1 & $\mathrm{BL} / \mathrm{Fe}$ II \\
\hline 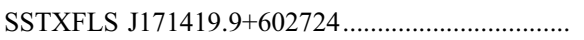 & 5.6 & 2.99 & $2 \mathrm{HZ}$ & Narrow UV lines \\
\hline 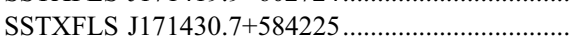 & 8.2 & 0.561 & $1 \mathrm{R}$ & $\mathrm{BL}^{\dagger}$ \\
\hline 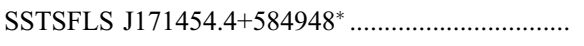 & 4.9 & 0.253 & Sbt & $\mathrm{BPT}$ \\
\hline SSTXFLS J171513.8+594638 & 5.0 & 0.248 & $1 \mathrm{R}$ & BL (radio-loud) \\
\hline 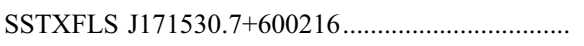 & 11.6 & 0.420 & 2 & {$[\mathrm{Ne} \mathrm{v}] 26$} \\
\hline 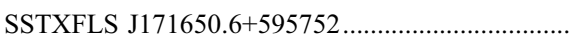 & 6.7 & 0.182 & 2 & BPT (composite) \\
\hline 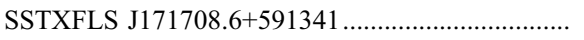 & 5.4 & 0.646 & 2 & {$[\mathrm{Ne} \mathrm{v}] 37$} \\
\hline 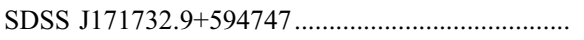 & 4.6 & 1.058 & 1 & $\mathrm{BL}$ \\
\hline 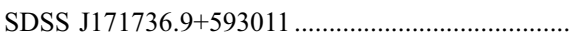 & 6.4 & 0.599 & 1 & BL \\
\hline SDSS J171747.4+593258 & 5.4 & 0.248 & 1 & BL \\
\hline 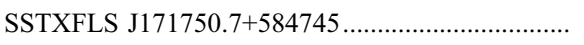 & 5.1 & $2.55 ?$ & $1 \mathrm{R} ?$ & BL? \\
\hline 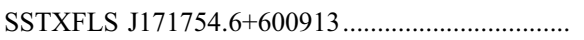 & 8.9 & 4.27 & $2 \mathrm{HZ}$ & Narrow UV lines \\
\hline 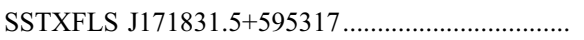 & 8.3 & 0.700 & 2 & {$[\mathrm{Ne} \mathrm{v}] 26$} \\
\hline 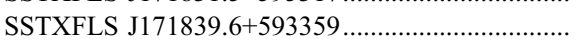 & 10.9 & 0.383 & 1 & BL (radio-loud) \\
\hline 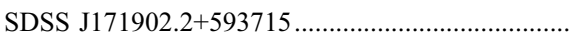 & 26.9 & 0.178 & 1 & $\mathrm{BL} / \mathrm{Fe}$ II \\
\hline 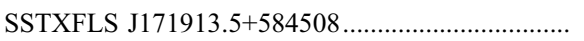 & 8.9 & 0.318 & 2 & {$[\mathrm{Ne} v] 12, \mathrm{BPT}$} \\
\hline 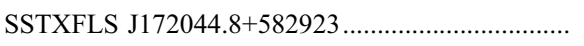 & 5.1 & 1.697 & $2 \mathrm{HZ}$ & Narrow UV lines \\
\hline 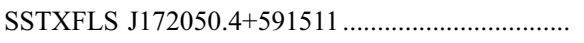 & 9.4 & 1.44 & $1 \mathrm{R}$ & $\mathrm{BL}$ \\
\hline 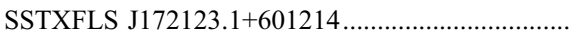 & 13.3 & 0.325 & 2 & {$[\mathrm{Ne} \mathrm{v}] 33, \mathrm{BPT}$} \\
\hline 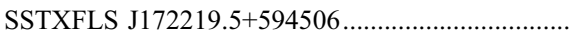 & 7.8 & 0.271 & 2 & $\mathrm{BPT}$ \\
\hline 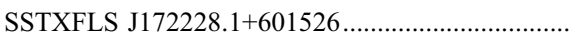 & 7.1 & 0.741 & 2 & {$[\mathrm{Ne} \mathrm{v}] 15$} \\
\hline SDSS J172238.7+585107 & 6.7 & 1.617 & 1 & $\mathrm{BL}$ \\
\hline 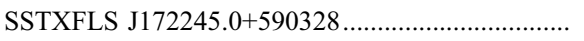 & 4.7 & 0.797 & 2 & {$[\mathrm{Ne} \mathrm{v}] 43$} \\
\hline SSTXFLS J172248.9+583256 & 5.6 & $0.84 \ddagger$ & $1 \mathrm{R}$ & $\mathrm{BL}$ \\
\hline 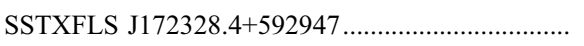 & 8.1 & $1.34 ?$ & $1 \mathrm{R}$ & $\mathrm{BL}$ \\
\hline 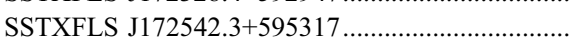 & 9.5 & 0.437 & $\mathrm{Sbt} / \mathrm{L}$ & Low ionization \\
\hline SDSS J172619.7+601600 & 6.6 & 0.925 & 1 & BL \\
\hline
\end{tabular}

Notes.-An asterisk indicates a spectrum from Papovich et al. (2006). A dagger indicates a classification based on the Papovich et al. spectrum; see Appendix A. A double dagger indicates that the optical/near-infrared redshift is uncertain, but a Spitzer mid-infrared spectrum shows silicate absorption at $z=0.84$ (M. Lacy et al. 2007, in preparation).

${ }^{a}$ LoBAL: Low-ionization broad absorption line.

study has found several objects that qualify as type 3 quasars (following Leipski et al. 2005), namely, galaxies with emissionline properties more typical of LINER or starburst galaxies than narrow-line AGNs. Leipski et al. find that $57 \%$ of their $I S O /$ 2MASS-selected AGNs have these optical spectra. MartínezSansigre et al. $(2005,2006)$ also deduce the presence of a significant population of obscured narrow-line AGNs from their mid-infrared/radio-selected objects, which show no emission lines in the rest-frame UV.

Our study has a lower fraction. Including objects with both starburst and ambiguous starburst/LINER spectra, we find a type 3 fraction of $\approx 9 \%$, which is, of course, an upper limit, as, apart from one case that is detected in the X-ray at the level expected for an AGN (SWIRE2 J021749.00-052306.9), we cannot prove the presence of AGNs in these objects. Thus, despite the inferred presence of hot dust, infrared spectroscopy or X-ray detection (at a level greater than expected for a starburst) is required to prove the AGN nature of these candidate type 3 objects. If they are reddened AGNs, the reddening may be in the host (e.g., Martínez-Sansigre et al. 2005; Rigby et al. 2006), although we cannot eliminate the possibility that nuclear dust is obscuring the narrow-line region or blocking the line of sight to the narrow-line region from the nucleus.

$\mathrm{X}$-ray surveys also find a population of AGNs that lack optical evidence for AGN activity: the X-ray-bright, optically normal galaxies (XBONGs; e.g., Comastri et al. 2002). A number of processes have been suggested for the lack of optical AGN activity in these objects, including host galaxy obscuration (Rigby et al. 
TABLE 4

BRIGHT AGNs in THE SWIRE XMM-LSS REGION

\begin{tabular}{|c|c|c|c|c|}
\hline Name & $\begin{array}{c}S_{24.0} \\
(\mathrm{mJy})\end{array}$ & Redshift & Class & Basis \\
\hline 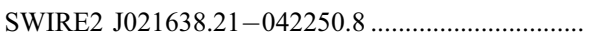 & 14.6 & 0.304 & 2 & BPT \\
\hline 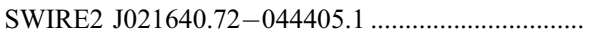 & 14.8 & 0.870 & 1 & Becker et al. (2001) \\
\hline 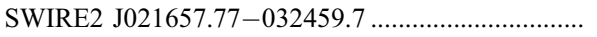 & 24.2 & 0.137 & 1 & $\mathrm{BL}$ \\
\hline 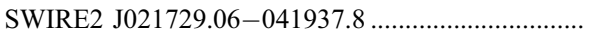 & 8.9 & 1.146 & $1 \mathrm{R}$ & $\mathrm{BL}$ \\
\hline 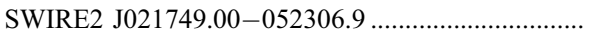 & 8.1 & $0.987 ?$ & $\mathrm{Sbt} / \mathrm{L} ?$ & Low ionization? \\
\hline 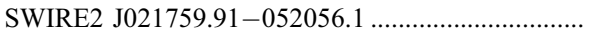 & 8.8 & 0.535 & 2 & {$[\mathrm{Ne} \mathrm{v}] 26$} \\
\hline 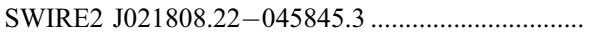 & 9.2 & 0.712 & 1 & Caccianiga et al. (2004) \\
\hline 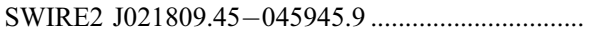 & 7.8 & 1.094 & 2 & {$[\mathrm{Ne} \mathrm{v}] 90$} \\
\hline 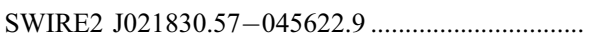 & 8.5 & 1.401 & 1 & Sharp et al. (2002) \\
\hline 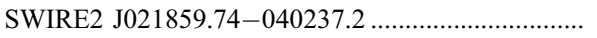 & 16.0 & 0.203 & 2 & BPT \\
\hline 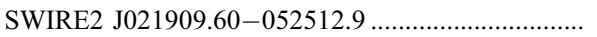 & 25.6 & 0.099 & 2 & BPT \\
\hline 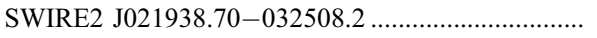 & 6.6 & 0.435 & 1 & Fe II \\
\hline 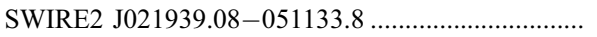 & 32.9 & 0.150 & 2 & {$[\mathrm{Ne} v] 9$} \\
\hline 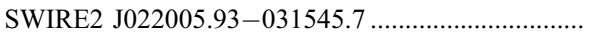 & 6.6 & 1.560 & 1 & $\mathrm{BL}$ \\
\hline 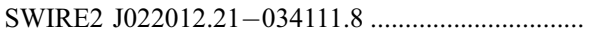 & 6.8 & 0.166 & 1 & BL \\
\hline 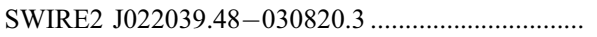 & 10.6 & 0.451 & 1 & BL \\
\hline 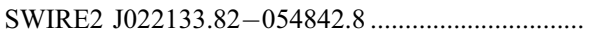 & 6.9 & 0.502 & 1 & $\mathrm{BL}$ \\
\hline 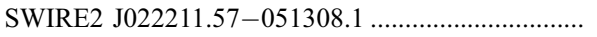 & 6.7 & 0.841 & 2 & {$[\mathrm{Ne} \mathrm{v}] 30$} \\
\hline 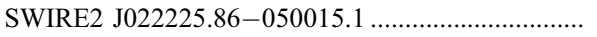 & 8.3 & 0.149 & 2 & BPT (composite) \\
\hline 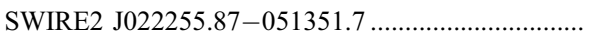 & 8.3 & 0.846 & 1 & $\mathrm{BL}$ \\
\hline 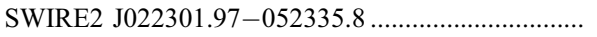 & 6.8 & $0.708 ?$ & $\mathrm{Sbt} / \mathrm{L} ?$ & Low ionization? \\
\hline 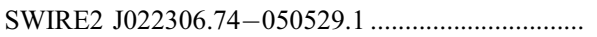 & 16.0 & 0.330 & 2 & BPT \\
\hline 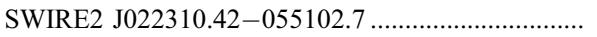 & 13.3 & 0.506 & 2 & {$[\mathrm{Ne} \mathrm{v}] 15$} \\
\hline 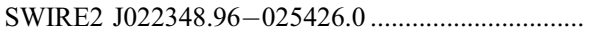 & 7.6 & 0.355 & $\mathrm{Sbt} / \mathrm{L}$ & Low ionization \\
\hline 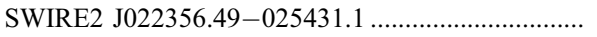 & 10.6 & 0.451 & 2 & {$[\mathrm{Ne} \mathrm{v}] 16$} \\
\hline 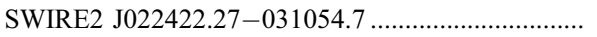 & 7.8 & 1.226 & 1 & BL \\
\hline 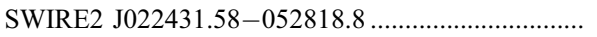 & 9.4 & 2.068 & 1 & $\mathrm{BL}$ \\
\hline 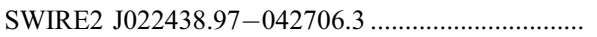 & 6.6 & 0.252 & 2 & BPT \\
\hline 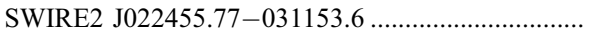 & 7.6 & 0.790 & $2 ?$ & {$[\mathrm{O} \mathrm{III}] /[\mathrm{O}$ II $]>1$} \\
\hline 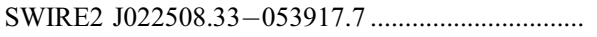 & 9.6 & 0.293 & 2 & BPT \\
\hline 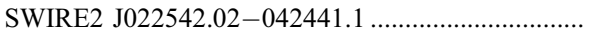 & 10.9 & 0.580 & 2 & {$[\mathrm{Ne} \mathrm{v}] 23$} \\
\hline 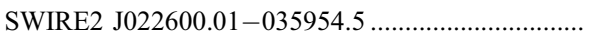 & 9.0 & $1.095 ?$ & $1 \mathrm{R}$ & $\mathrm{BL}$ \\
\hline 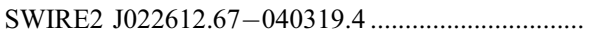 & 7.7 & 0.330 & 2 & BPT \\
\hline 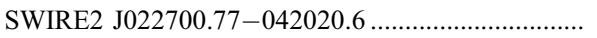 & 27.1 & 0.053 & 1 & $\mathrm{Fe}$ II \\
\hline
\end{tabular}
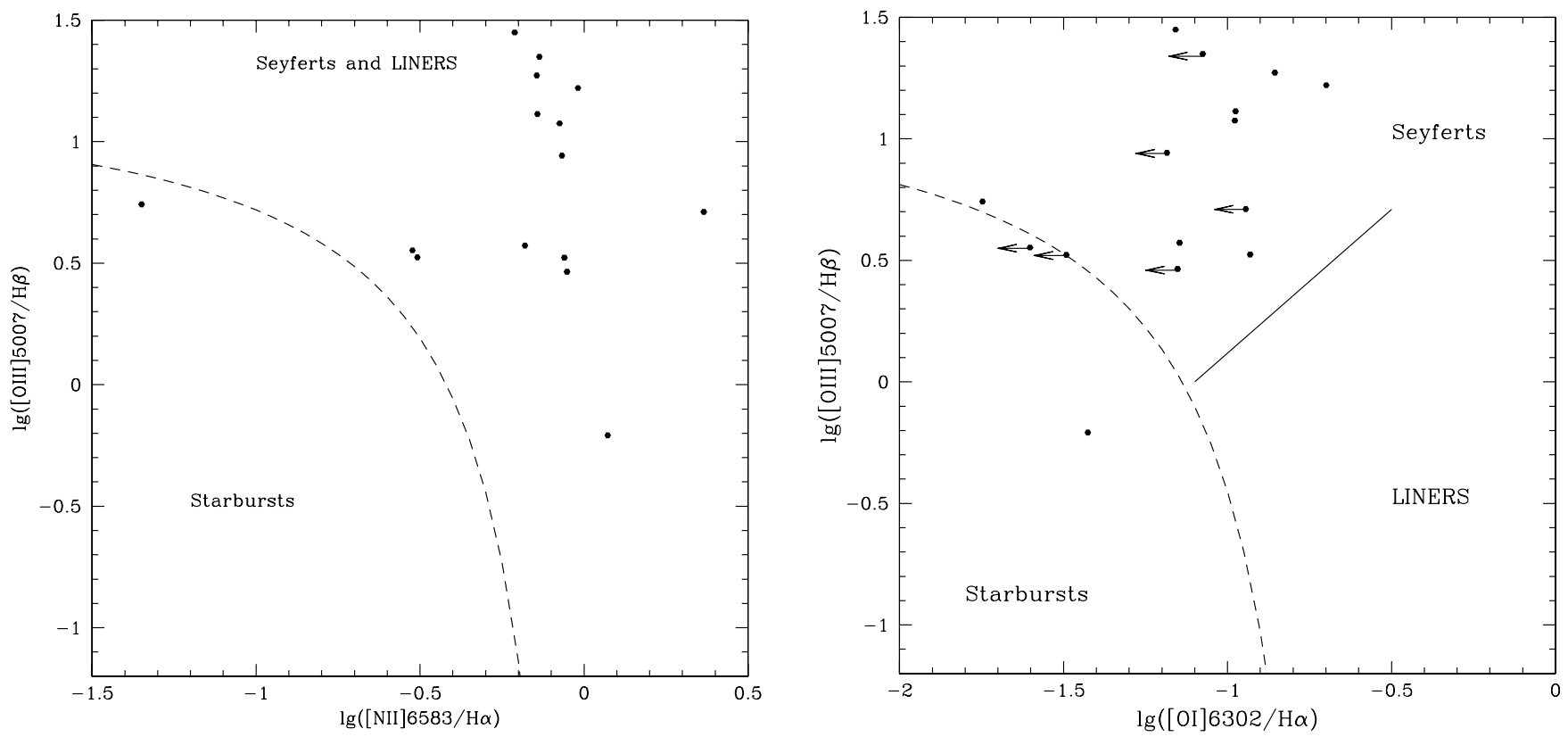

FIg. 4.-Two diagnostic (BPT) diagrams for our lower redshift emission-line galaxies that have H $\alpha$ in the optical window. The dividing lines are from Kewley et al. (2006). Most of our objects plot among the AGNs; there are no LINERs in the sample. 


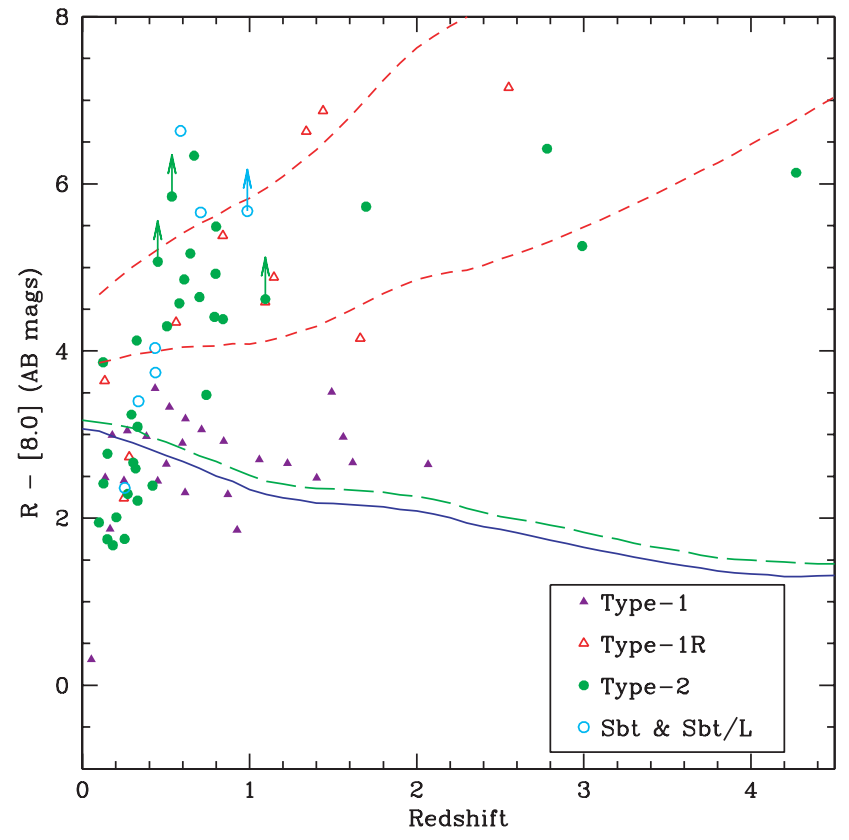

FIG. 5.-Optical-to-infrared color for our quasar samples. Type 3 objects that lack redshifts have been assigned a redshift of 1.50. The color loci of two of the quasar SEDs of Richards et al. (2006) are plotted as lines. The blue solid line shows the mean quasar SED, and the green dashed line shows the IR-luminous SED. The red dashed lines indicate the colors of the mean quasar SED reddened by the Small Magellanic Cloud extinction law of Pei (1992). The lower line corresponds to a rest-frame reddening of $E(B-V)=0.32$, and the upper line corresponds to 0.65 . The dispersion in optical-to-mid-infrared color of the quasars of Richards et al. (2006) is $\approx \pm 0.3$ mag.

2006), radiatively inefficient accretion (Yuan \& Narayan 2004), or dilution of weak emission lines by host galaxy light (Moran et al. 2002; Eckart et al. 2006). The discovery of [N v] $\lambda 3426$ emission in a stacked spectrum of XBONGs made by Eckart et al. (2006) favors dilution and/or extinction over a fundamental difference in accretion mode. The same may well be true if our type 3

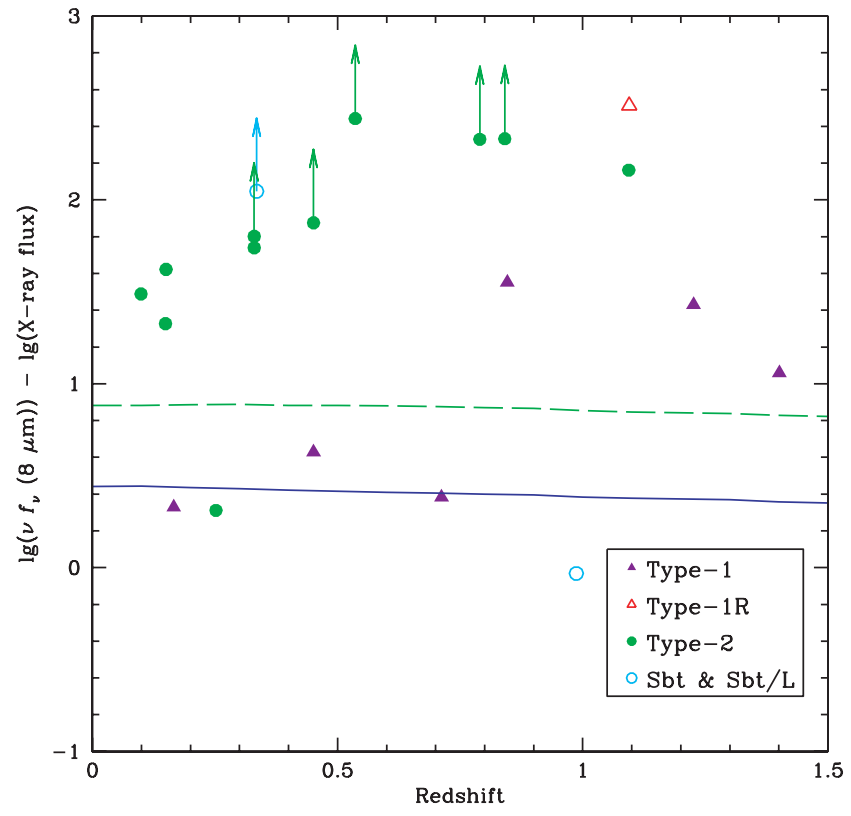

FIG. 6.-Observed X-ray (0.2-12 keV) flux vs. observed $8.0 \mu \mathrm{m}$ infrared flux ratios for objects in archival XMM-Newton fields. The blue solid line shows the mean quasar SED of Richards et al. (2006) extrapolated from the soft X-ray, assuming a photon index of 1.8 ; the green dashed line indicates the same for the IR-luminous SED.

quasars really are AGNs, particularly any that are composite starburst-AGN systems with strong star formation activity in the host galaxies. In particular, objects that are classified on the basis of line ratios in the BPT diagram may have their AGN line emission overwhelmed by the starburst component, which is likely to dominate the overall energetics of the galaxy.

Dilution of AGN signatures by host galaxy emission may help to explain why our type 3 fraction is lower than those of Leipski et al. (2005), whose objects are mostly of lower redshift and luminosity, where host galaxy contamination is more of an issue.

TABLE 5

XMM-Newton Detections of AGNs in the SWIRE Field

\begin{tabular}{|c|c|c|c|c|c|c|c|c|}
\hline Object Name & $z$ & Optical Class & Obs ID & Exposure Time & Camera & Counts & Source & $\begin{array}{c}S_{0.2-12 \mathrm{keV}} \\
\left(10^{-14} \mathrm{ergs}^{-1} \mathrm{~cm}^{-2}\right)\end{array}$ \\
\hline SWIRE2 J021749.00-052306.9 .................... & $0.987 ?$ & $\mathrm{Sbt} / \mathrm{L} ?$ & 0112370701 & 47648 & pn & 20050 & 2XMM & 178 \\
\hline SWIRE2 J021759.91-052056.1 .................... & 0.535 & 2 & 0112370801 & 47330 & pn & $\lesssim 50$ & PPS & $\lesssim 0.4$ \\
\hline SWIRE2 J021808.22-045845.3 .................... & 0.712 & 1 & 0112370101 & 57631 & pn & 14500 & 1XMM & 104 \\
\hline SWIRE2 J021809.45-045945.9 .................... & 1.094 & 2 & 0112370101 & 57631 & pn & 100 & PPS & 0.75 \\
\hline SWIRE2 J021830.57-045622.9 .................. & 1.401 & 1 & 0112370101 & 57631 & pn & 3100 & 1XMM & 22 \\
\hline SWIRE2 J021909.60-052512.9 .................... & 0.099 & 2 & 0112370801 & 47330 & pn & 300 & 2XMM & 2.6 \\
\hline SWIRE2 J021939.08-051133.8 .................... & 0.150 & 2 & 0112370801 & 47330 & pn & 601 & 1XMM & 5.3 \\
\hline SWIRE2 J022012.21-034111.8 .................... & 0.166 & 1 & 0037982601 & 11592 & pn & 1740 & 2XMM & 63 \\
\hline SWIRE2 J022039.48-030820.3 ................... & 0.451 & 1 & 0037982701 & 14892 & $\mathrm{pn}$ & 1100 & 2XMM & 31 \\
\hline SWIRE2 J022211.57-051308.1 .................... & 0.841 & 2 & 0111110501 & 20056 & pn & $\lesssim 20$ & PPS & $\lesssim 0.4$ \\
\hline SWIRE2 J022225.86-050015.1 ................... & 0.149 & 2 & 0111110501 & 20056 & MOS2 & 30 & PPS & 2.0 \\
\hline SWIRE2 J022255.87-051351.7 ................... & 0.846 & 1 & 0111110501 & 20056 & pn & 20 & 1XMM & 22 \\
\hline SWIRE2 J022306.74-050529.1 .................... & 0.330 & 2 & 0111110501 & 20056 & $\mathrm{pn}$ & 46 & 1XMM & 0.95 \\
\hline SWIRE2 J022348.96-025426.0 ................... & 0.355 & $\mathrm{Sbt} / \mathrm{L}$ & 0037981601 & 13319 & pn & $\lesssim 26$ & PPS & $\lesssim 0.8$ \\
\hline SWIRE2 J022356.49-025431.1 ................... & 0.451 & 2 & 0037981601 & 13319 & $\mathrm{pn}$ & $\lesssim 26$ & PPS & $\lesssim 0.8$ \\
\hline SWIRE2 J022422.27-031054.7 ................... & 1.226 & 1 & 0037981201 & 8420 & pn & 170 & 2XMM & 8.4 \\
\hline SWIRE2 J022438.97-042706.3 ................... & 0.252 & 2 & 0112680501 & 10040 & $\mathrm{pn}$ & 1600 & 2XMM & 30 \\
\hline SWIRE2 J022455.77-031153.6 ................... & 0.790 & $2 ?$ & 0037981301 & 5702 & $\mathrm{pn}$ & $\lesssim 10$ & PPS & $\lesssim 0.8$ \\
\hline SWIRE2 J022542.02-042441.1 .................. & 0.580 & 2 & 0112681001 & 41946 & pn & $\lesssim 40$ & PPS & $\lesssim 0.4$ \\
\hline SWIRE2 J022600.01-035954.5 ................... & $1.095 ?$ & $1 \mathrm{R}$ & 0112680201 & 13675 & pn & 33 & 2XMM & 1.0 \\
\hline SWIRE2 J022612.67-040319.4 ................... & 0.330 & 2 & 0112680201 & 13675 & $\mathrm{pn}$ & $\lesssim 27$ & PPS & $\lesssim 0.8$ \\
\hline
\end{tabular}




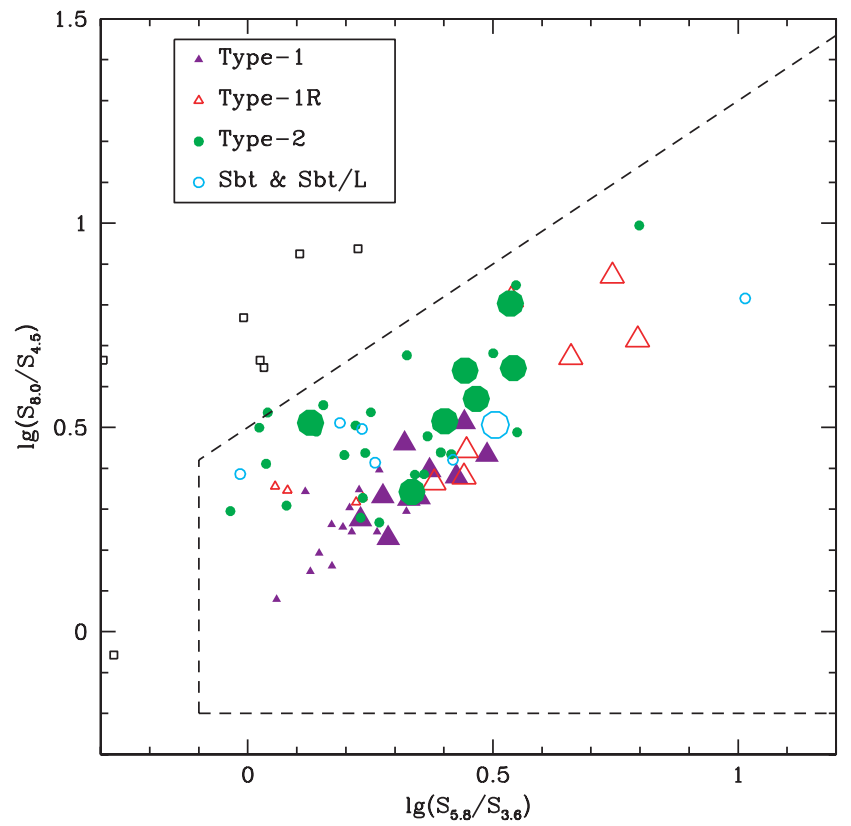

FIG. 7.-Enlarged view of the AGN wedge of Fig. 1. Large symbols represent $z \geq 0.8$ objects, and small symbols represent those at $z<0.8$. Open squares represent the objects from the sample of Table 6 that have AGN-like optical emissionline ratios but non-AGN IRAC colors.

Our type 3 fraction may be smaller than that of Martínez-Sansigre et al. $(2005,2006)$ due to our order of magnitude brighter flux limit at $24 \mu \mathrm{m}$ allowing us to find more objects with intrinsically faint or lightly reddened $[E(B-V) \sim 0.1-1]$ narrow lines. Certainly, it is apparent from our spectroscopic campaign that even objects with $24 \mu \mathrm{m}$ fluxes of several mJy can require $1.5 \mathrm{hr}$ of integration on a $5 \mathrm{~m}$ telescope to see emission lines.

\subsection{Reliability and Effectiveness of Mid-Infrared Selection of $A G N S$}

The IRAC color technique is clearly effective at selecting AGNs, at least for objects with fluxes of a few mJy at $24 \mu \mathrm{m}$. We have been able to unambiguously classify most of our objects as AGNs based on their optical spectra. Contaminants are certainly possible, however. At moderate redshifts starbursts will be able to move into the AGN wedge if they have large amounts of warm dust, and at high redshifts the $1.6 \mu \mathrm{m}$ bump of stellar SEDs can lead to IRAC colors that mimic those of AGNs. We can state that for this sample of 77 bright $24 \mu \mathrm{m}$ sources, only seven lack obvious optical signatures of AGN activity, and of these, one is detected in an XMM-Newton field, which implies a contamination rate of $<6$ out of $77(8 \%)$.

Of course, the selection of bright $24 \mu \mathrm{m}$ sources itself produces a large fraction of AGNs, as demonstrated by Brand et al. (2006). In particular, when combined with optical photometric criteria, a sample containing a large fraction of AGNs can be made by simply rejecting the optically bright objects. To investigate how effective such a cut is, and to take a first look at the completeness of our AGN sample, we have examined the brightest 50 sources in the XFLS $24 \mu \mathrm{m}$ list (Table 6). This sample contains 29 objects that are classified as AGNs according to the criteria of $\S 3$. As the starbursts are also concentrated at lower redshifts, this qualitatively confirms the result of Brand et al. (2006), namely, that the mid-infrared luminosity density is dominated by AGNs at millijansky flux levels. Objects with faint optical identifications are certainly more likely to be AGNs; no object with
$R>21$ is classified as a starburst or LINER, but apart from that division there is a wide range in magnitudes $(R \approx 17-20)$ over which AGNs and starbursts/LINERs overlap, particularly if no optical morphology criterion is used and thus quasars are included.

This sample of the 50 brightest $24 \mu \mathrm{m}$ sources also allows us to take a first look at how complete the AGN selection using IRAC colors is. Interestingly, we find that seven objects outside the IRAC color-based AGN selection show AGN-like emissionline ratios in a BPT diagram, although these are nearly all close to the AGN-starburst transition region (Fig. 8). Their positions in the IRAC color-color plot are shown in Figure 7. In six of these cases IRAC $8.0 \mu \mathrm{m}$ band flux is very high, pushing the objects into the star-forming galaxy region of the color-color plot of Figure 1. The remaining object (SSTXFLS J171150.2+590041) shows pure Seyfert emission-line ratios but has only a weak mid-infrared excess and is in the part of Figure 1 dominated by quiescent galaxies. This suggests that in some cases the signature of AGN activity in the mid-infrared can be overwhelmed by strong star formation activity, at least at $z \lesssim 0.3$, where the PAH features can still contribute to the IRAC channel 4 flux. It may also indicate that much star formation activity (particularly any close to the nucleus) may be more obscured by dust than the narrowline region of an AGN in the same galaxy. An AGN selection with the diagonal cut of the wedge raised by $\approx+0.5$ in $\log \left(S_{8.0} / S_{5.8}\right)$ would therefore increase the completeness of the AGN selection at low redshifts, although at the risk of reducing reliability.

\section{THE RATIO OF OBSCURED TO UNOBSCURED AGNs}

\subsection{Redshift and Luminosity Distributions}

Figure 9 shows the redshift histograms for our various classes of objects compared to the redshift distribution of the sample as a whole. Figure 10 shows the corresponding luminosity distributions of our objects. We calculate luminosities at a rest wavelength of $5 \mu \mathrm{m}$. This region of the continuum is free from features and can be calculated by interpolation between measurements over our full range of redshifts. The most heavily reddened AGNs will, however, still be significantly extincted at this wavelength, which is a possible bias in Figure 10.

As previously discussed, there are strong selection effects favoring the selection of low-z, high-luminosity AGNs in our sample. Therefore, we have made no attempt to investigate in detail the ratio of obscured to unobscured AGNs as a function of redshift or luminosity to compare with, for example, the receding torus model of Lawrence (1991).

Overall, the breakdown into optical spectral types is as follows: $33 \%$ (25 out of 77 ) normal type $1,14 \%$ (11 out of 77$)$ reddened type $1,44 \%$ (34 out of 77 ) type 2 (including $2 \mathrm{HZ}$ ), and $9 \%$ ( 7 out of 77) starbursts and ambiguous starbursts/LINERs. Defining an obscured quasar as anything with $E(B-V) \gtrsim 0.3$ in the rest frame and an AGN nature confirmed through optical emission lines or $\mathrm{X}$-rays (the type $1 \mathrm{R}$ objects, the type 2 objects, and the single X-ray-detected starburst/LINER), we obtain a ratio of obscured to unobscured objects of $1.8: 1$. If we assume that the other six starburst and starburst/LINER objects also contain obscured AGNs, this increases to $2.1: 1$.

The fraction of type $1 \mathrm{R}$ objects is higher than that found by Brown et al. (2006) in their $24 \mu \mathrm{m}$-selected sample. Brown et al. find that $20 \%$ of their broad-line objects are classified as reddened type 1 objects, compared to a type 1R/(type 1+type 1R) fraction of $30 \%$ (11 out of 36) in our sample. Our deeper spectroscopy limit ( $R \sim 23$ compared to $R<21.7$ ) is probably responsible for this difference. Two of our type 1R quasars have $R<21.7$, and a third 
TABLE 6

The 50 Brightest Extragalactic $24 \mu \mathrm{m}$ Sources in the XFLS with Four-Band IRAC Coverage

\begin{tabular}{|c|c|c|c|c|c|c|c|}
\hline Name & $\begin{array}{c}S_{24} \\
(\mathrm{mJy})\end{array}$ & $R$ & Spectrum $^{\mathrm{a}}$ & Redshift & Class & Basis & IRAC-selected AGN? \\
\hline 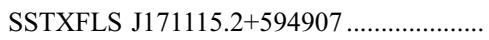 & 9.41 & 20.97 & L06 & 0.587 & Sbt & Strong Balmer lines & Yes \\
\hline 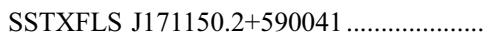 & 10.88 & 17.97 & P06 & 0.060 & 2 & $\mathrm{BPT}$ & No \\
\hline 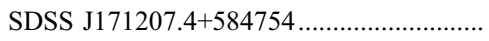 & 13.34 & 17.76 & L06 & 0.269 & 1 & $\mathrm{Fe}$ II & Yes \\
\hline 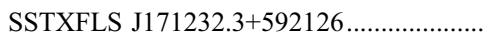 & 8.47 & 17.56 & P06 & 0.21 & $\mathrm{Sbt} / \mathrm{L}$ & Low ionization & No \\
\hline 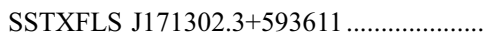 & 11.83 & 21.50 & L06 & 0.668 & 2 & {$[\mathrm{Ne} v] 33$} & Yes \\
\hline SSTXFLS J171313.9+603146 ....................... & 10.49 & 18.31 & L06 & 0.105 & 2 & BPT & Yes \\
\hline SSTXFLS J171325.1+590531 ….................. & 9.43 & 18.33 & L06 & 0.126 & 2 & BPT & Yes \\
\hline SSTXFLS J171335.1+584756 ...................... & 23.66 & 17.56 & L06 & 0.133 & $1 \mathrm{R}$ & $\mathrm{Fe}$ II & Yes \\
\hline 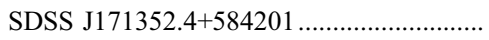 & 23.90 & 17.53 & SDSS & 0.521 & 1 & $\mathrm{BL} / \mathrm{Fe}$ II & Yes \\
\hline 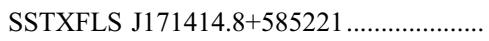 & 8.97 & 18.70 & P06 & 0.167 & Sbt & BPT & No \\
\hline SSTXFLS J171430.7+584225 ....................... & 8.24 & 19.72 & L06 & 0.561 & $1 \mathrm{R}$ & BL & Yes \\
\hline 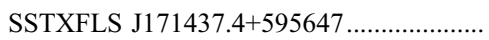 & 11.60 & 18.75 & P06 & 0.196 & 2 & BPT (composite) & No \\
\hline 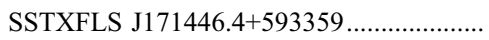 & 7.37 & 17.67 & P06 & 0.129 & $\mathrm{Sbt} / \mathrm{L}$ & Low ionization & No \\
\hline SSTXFLS J171530.7+600216 & 11.55 & 18.82 & L06 & 0.420 & 2 & {$[\mathrm{Ne} v] 26$} & Yes \\
\hline 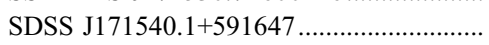 & 6.88 & 17.43 & SDSS & 0.116 & $\mathrm{Sbt} / \mathrm{L}$ & Low ionization & No \\
\hline 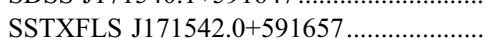 & 26.33 & 17.50 & P06 & 0.116 & Sbt & BPT & No \\
\hline SSTXFLS J171544.0+600835 & 6.86 & 17.07 & $\mathrm{~L}^{*} 6^{*}$ & 0.157 & 2 & BPT & No \\
\hline SDSS J171607.2+591456 & 34.57 & 16.27 & SDSS & 0.054 & Sbt & BPT & No \\
\hline SDSS J171614.4+595423 ……...................... & 8.41 & 17.39 & SDSS & 0.153 & Sbt & BPT & No \\
\hline SDSS J171630.1+601423 ............................. & 8.35 & 17.82 & SDSS & 0.107 & Sbt & BPT & No \\
\hline SSTXFLS J171634.0+601443 $\ldots \ldots \ldots \ldots \ldots \ldots \ldots$ & 10.76 & 17.04 & P06 & 0.108 & Sbt & $\mathrm{BPT}$ & No \\
\hline SDSS J171635.9+601436 & 7.66 & 17.07 & SDSS & 0.106 & Sbt & BPT & No \\
\hline 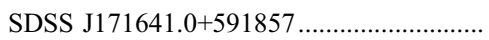 & 20.88 & 16.41 & SDSS & 0.056 & Sbt & $\mathrm{BPT}$ & No \\
\hline SSTXFLS J171650.5+595751 ….................... & 6.69 & 18.81 & L06 & 0.182 & 2 & BPT (composite) & Yes \\
\hline SSTXFLS J171711.1+602710 & 9.43 & 17.88 & L06* & 0.110 & $\mathrm{Sbt} / \mathrm{L}$ & Low ionization & No \\
\hline SSTXFLS J171744.1+583848 ........................ & 13.92 & 17.73 & P06 & 0.066 & $\mathrm{~L}$ & BPT & No \\
\hline SSTXFLS J171754.6+600913 ….................... & 8.94 & 21.80 & L06 & 4.27 & $2 \mathrm{HZ}$ & Narrow UV lines & Yes \\
\hline 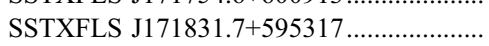 & 8.27 & 20.56 & L06 & 0.700 & 2 & {$[\mathrm{Ne} v] 26$} & Yes \\
\hline 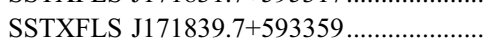 & 13.22 & 17.99 & L06 & 0.383 & 1 & $\mathrm{BL}$ & Yes \\
\hline 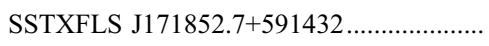 & 13.92 & 19.23 & P06 & 0.322 & $2 / \mathrm{L}$ & $\mathrm{BPT}$ & No \\
\hline SSTXFLS J171902.2+593715 ........................... & 26.91 & 17.10 & L06 & 0.178 & 1 & $\mathrm{BL} / \mathrm{Fe}$ II & Yes \\
\hline 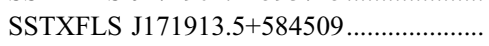 & 8.90 & 19.00 & L06 & 0.318 & 2 & {$[\mathrm{Ne} v] 12, \mathrm{BPT}$} & Yes \\
\hline 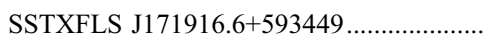 & 17.40 & 17.91 & P06 & 0.166 & $2 / \mathrm{L}$ & BPT & No \\
\hline 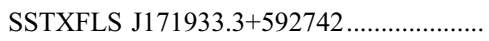 & 7.53 & 17.71 & P06 & 0.139 & 2 & BPT (composite) & No \\
\hline SDSS J171944.8+595705 & 6.73 & 16.66 & SDSS & 0.069 & Sbt & $\mathrm{BPT}$ & No \\
\hline 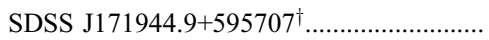 & 7.44 & 16.66 & SDSS & 0.069 & Sbt & BPT & No \\
\hline SSTXFLS J172028.9+584749 _..................... & 9.57 & 16.74 & L06* & 0.127 & 2 & BPT & No \\
\hline SDSS J172043.2+584026 & 9.48 & 17.08 & SDSS & 0.125 & Sbt & BPT & No \\
\hline SSTXFLS J172050.5+591512 …................... & 9.41 & 21.62 & L06 & 1.44 & $1 \mathrm{R}$ & $\mathrm{BL}$ & Yes \\
\hline 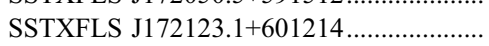 & 13.34 & 18.78 & L06 & 0.325 & 2 & {$[\mathrm{Ne} v] 33, \mathrm{BPT}$} & Yes \\
\hline 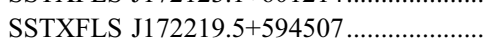 & 7.76 & 18.71 & L06 & 0.272 & 2 & BPT & Yes \\
\hline SSTXFLS J172220.2+590949 _...................... & 8.78 & 17.58 & P06 & 0.179 & Sbt & BPT & No \\
\hline SSTXFLS J172228.0+601525 ..................... & 7.12 & 19.85 & L06 & 0.741 & 2 & {$[\mathrm{Ne} \mathrm{v}] 15$} & Yes \\
\hline 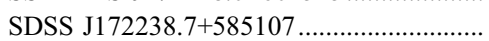 & 6.73 & 18.17 & SDSS & 1.617 & 1 & $\mathrm{BL}$ & Yes \\
\hline 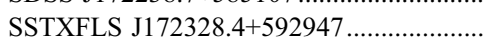 & 8.10 & 22.15 & L06 & $1.34 ?$ & $1 \mathrm{R}$ & BL & Yes \\
\hline 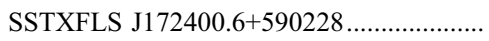 & 13.34 & 17.99 & P06 & 0.178 & Sbt & BPT & No \\
\hline 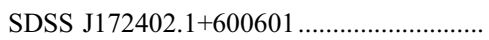 & 7.76 & 17.12 & SDSS & 0.156 & $\mathrm{Sbt} / \mathrm{L}$ & Low ionization & No \\
\hline SSTXFLS J172508.8+591016 ....................... & 9.40 & 17.78 & P06 & 0.164 & Sbt & BPT & No \\
\hline 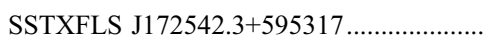 & 9.45 & 20.03 & L06 & 0.437 & $\mathrm{Sbt} / \mathrm{L}$ & Low ionization & Yes \\
\hline 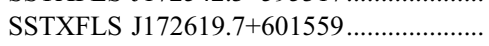 & 6.57 & 17.13 & L06 & 0.925 & 1 & $\mathrm{BL}$ & Yes \\
\hline
\end{tabular}

Notes.-An asterisk indicates additional spectra obtained using COSMIC on the Palomar 200 inch telescope, 2005 June 27-28. A dagger indicates parts of a single galaxy.

a Provenance of spectra: L06, this paper; P06, Papovich et al. (2006); and SDSS, SDSS Data Release 4.

has $R=21.6$; excluding these would reduce our type $1 \mathrm{R}$ fraction to $25 \%$.

Before we can relate these ratios to an estimate of how much supermassive black hole growth is obscured, we need to bear in mind the intrinsic variations in quasar SEDs. Selection in the midinfrared will inevitably mean our sample is biased in favor of objects whose intrinsic SEDs (before reddening of the quasar nucleus) are particularly bright in the mid-infrared, e.g., because of an un- usually large dust-covering factor. For type 1 and type 1R objects we have good evidence that this is not a large effect. Brown et al. (2006) find that the space density of their infrared-selected quasars is very similar to that of optically selected samples, consistent with the small systematic variations seen in the quasar SEDs of Richards et al. (2006) and Hatziminaoglou et al. (2005) between optically bright and infrared-bright quasars. Although our type 1 quasars at $z>1$ are redder in $R-[8.0]$ color than the Richards 


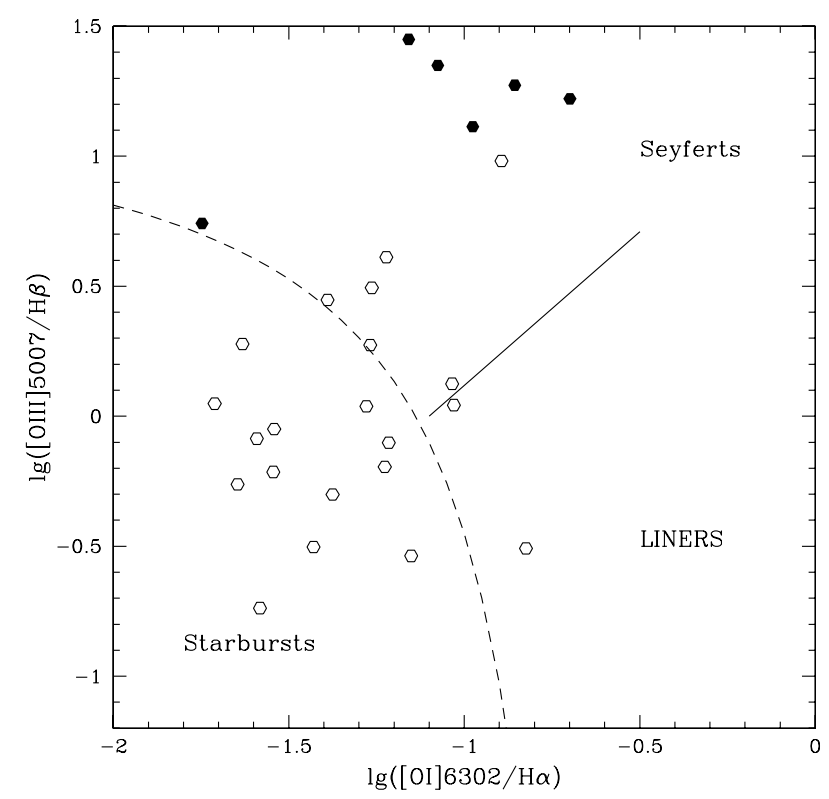

Fig. 8.- $[\mathrm{O} \mathrm{I}] / \mathrm{H} \alpha$ vs. $[\mathrm{O}$ III $] / \mathrm{H} \beta$ BPT diagram for the objects of Table 6 (the 50 brightest $24 \mu \mathrm{m}$ sources, regardless of IRAC colors) with [O III] detections. Objects falling outside of the IRAC AGN color selection are shown as open symbols, and those within it are shown as filled symbols.
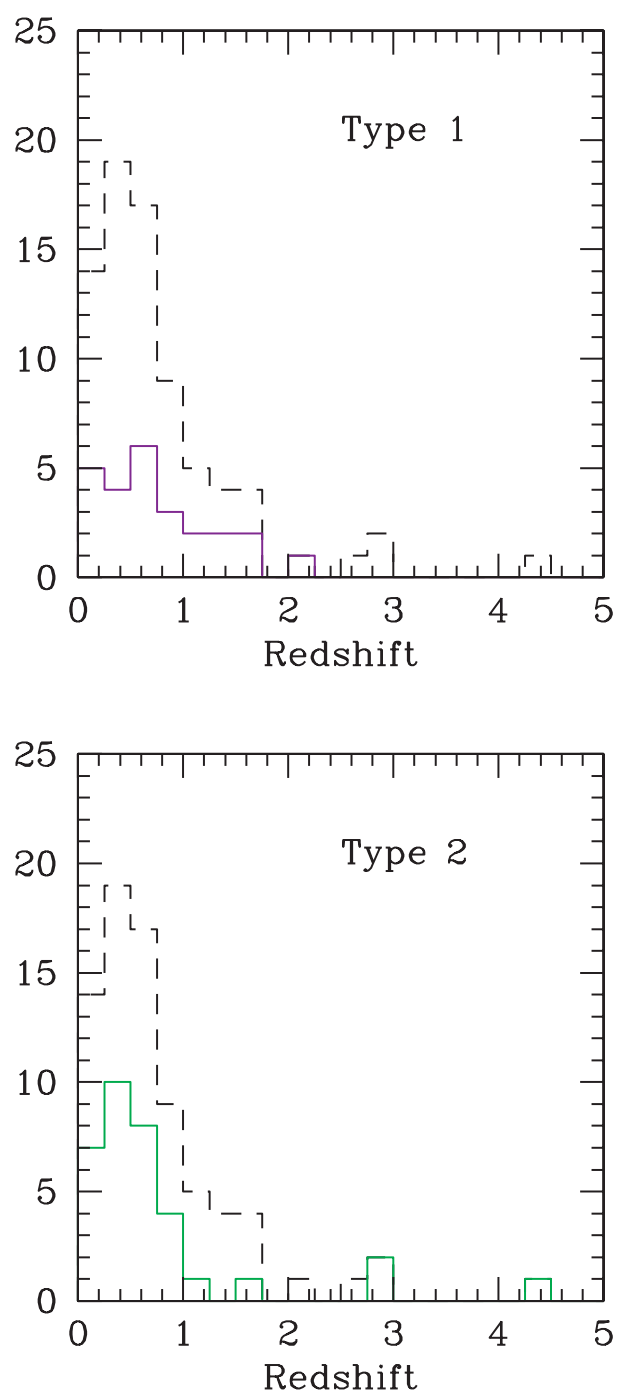

et al. template color in Figure 5, most are close to the template, and several at $z<1$ are bluer. For the more heavily obscured objects there are two arguments that can be brought to bear. First, even in type 1 quasars about $40 \%$ of the UV emission is reprocessed by dust (Richards et al. 2006). Even if the obscured quasars have dust-covering factors near unity, their dust emission would only increase by a factor of $\sim 2$ (and they would be significantly extincted in the mid-infrared if that were the case, leading to their infrared luminosities being underestimated). Second, the majority of the obscured quasars possess luminous narrow emission lines, showing that a significant fraction of lines of sight from the nucleus are able to escape to ionize the narrow-line gas. Furthermore, in the case of radio-loud objects, in which the radiolobe luminosity provides an independent measure of the AGN luminosity, Haas et al. (2004b) find that radio-loud quasars and their type 2 equivalents, radio galaxies, show a statistically identical ratio of radio to $10-1000 \mu \mathrm{m}$ infrared luminosity, indicating that the obscured population has a dust-covering factor similar to that of the unobscured population, as expected from orientation-based unified schemes (Antonucci 1993). We are thus confident that mid-infrared selection does not significantly bias an AGN sample toward objects with unusually high dust-covering factors, and so we are fairly confident that our AGN sample is a fair proxy for one selected on the basis of bolometric flux. We can thus safely
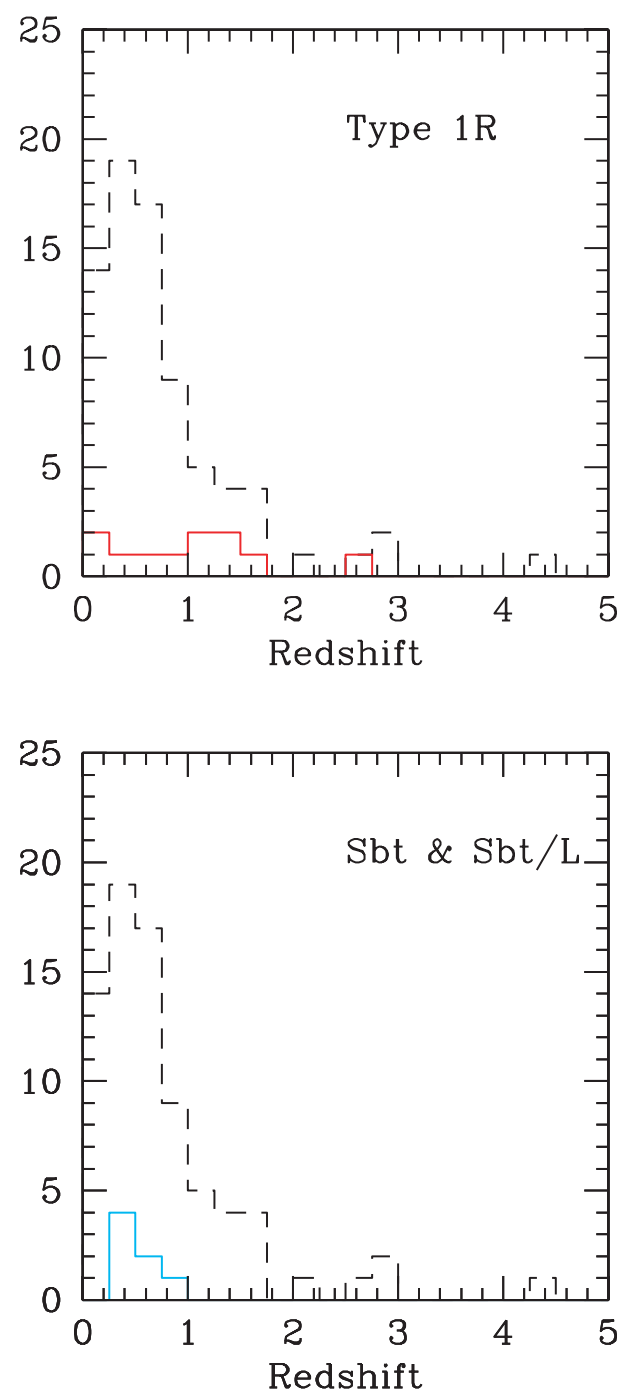

Fig. 9.- Redshift distribution for our different classes of objects. The total distribution for the whole sample is shown as the dotted line, and the solid line shows the distribution by type. 

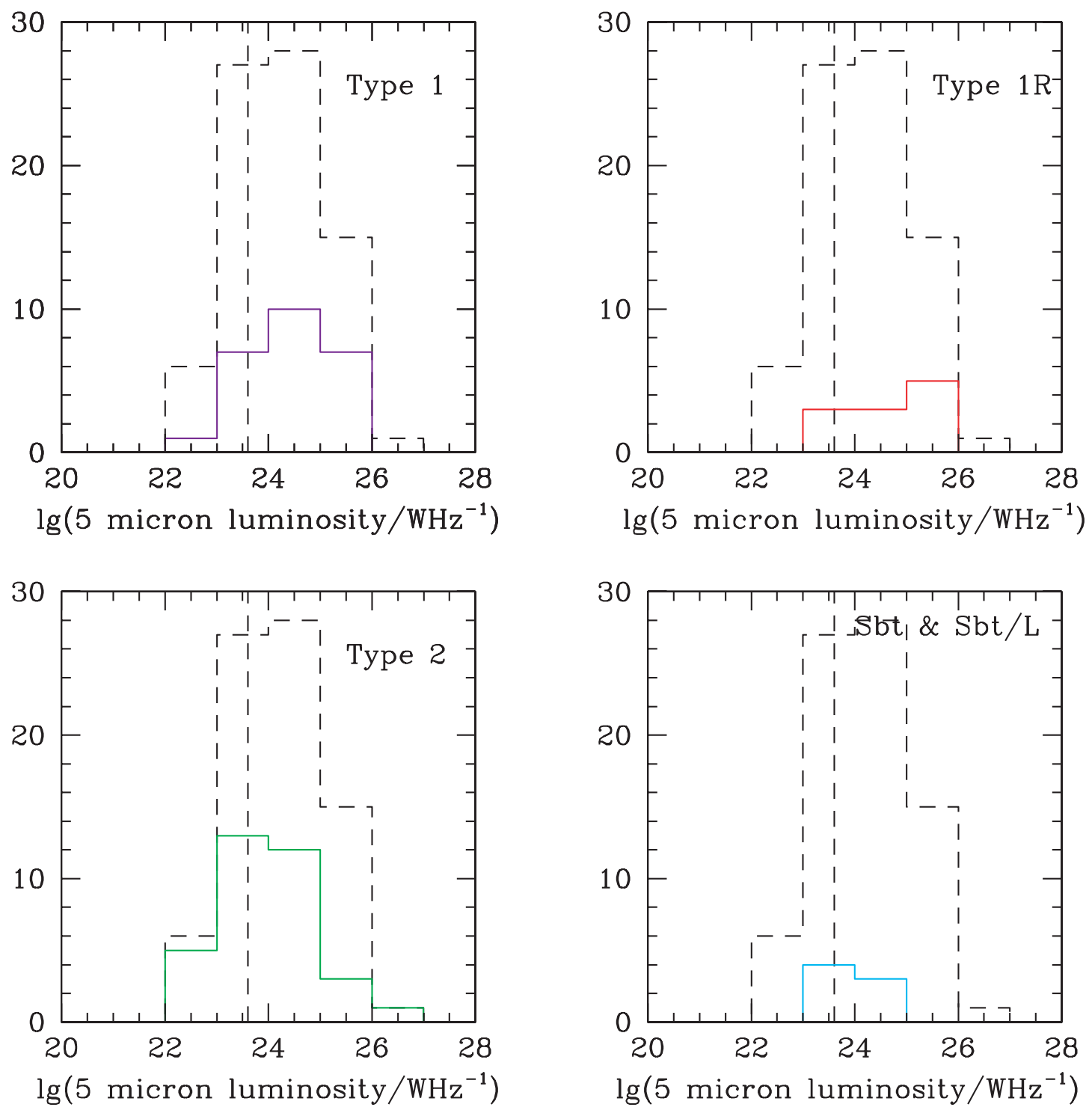

FIG. 10.-Same as Fig. 9, but for rest-frame luminosity at $5 \mu \mathrm{m}$. The vertical dashed line is at the approximate division between Seyfert and quasar luminosities.

conclude that $\sim 67 \%$ of black hole growth in the redshift and luminosity range probed in this study is obscured by $E(B-V) \gtrsim 0.3$.

\subsection{Comparison to Other Spitzer-Selected Samples}

Mid-infrared selection of obscured AGNs has been successfully performed by several other groups. Stern et al. (2005) use Hectospec data on the Bootes field to study AGNs selected using a mid-infrared color cut similar to that in Figure 1 but with different axes. Plotting the objects of this paper onto the selection of Stern et al., we find that only a few obscured AGNs found within our mid-infrared color criteria are missed by their color selection. They find an $\approx 3: 1$ ratio of obscured to unobscured AGNs, somewhat higher than found in this study, which is $\approx 2: 1$. However, as Richards et al. (2006) point out, their result relies on a power-law interpolation of the quasar SED between the midinfrared and optical, which is not quite correct (see, e.g., the variation of $R-$ [8.0] color in Fig. 5), so their obscured AGN fraction may be overestimated. Alonso-Herrero et al. (2006) use another, slightly different mid-infrared selection, based on the presence of a power-law mid-infrared SED, to find AGNs in the Chandra Deep Field South. Due to this rather more complicated selection criterion it is hard to assess how many objects in the color-selected
AGN samples would satisfy the selection criteria of AlonsoHerrero et al., but most of them are likely to, as our color criterion is very similar to a power-law selection. Their study complements the study of this paper very well, as their $24 \mu \mathrm{m}$ fluxes range from $\approx 0.05$ to $3 \mathrm{mJy}$ compared to our range of $\approx 4.4-20 \mathrm{mJy}$. Their median redshift of $\sim 1.4$ (based mostly on photometric redshifts) compares with a median of 0.6 for the combined spectroscopic XFLS and SWIRE samples. Thus, their objects are typically of similar or only slightly lower luminosity than the objects in this study. The fact that they find a similar ratio of obscured to unobscured AGNs of $\approx 2: 1$ (based on SED fitting) is therefore consistent with no strong dependence on this ratio on redshift in the range $\sim 0.5-2$.

Martínez-Sansigre et al. $(2005,2006)$ use a different approach to selecting their obscured AGNs. By searching for $24 \mu \mathrm{m}$ sources with radio emission in excess of the radio-infrared correlation they are able to isolate obscured AGNs irrespective of their midinfrared SED shape. Although the properties of these radiointermediate objects may be different from the predominately radio-quiet populations found from the mid-infrared color selection, the ratio of obscured to unobscured objects is similar, in the range $1-3: 1$. 
In the X-ray, Alonso-Herrero et al. (2006), Donley et al. (2005), and Polletta et al. (2006) find a significant population (40\%-60\%) of Spitzer-selected AGNs that are highly obscured in the X-ray. In particular, many Spitzer-selected AGNs in the samples of AlonsoHerrero et al. and Donley et al. are missing from even the $\sim 1 \mathrm{Ms}$ exposures of the Chandra Deep Fields. Twenty-one of the objects in our samples overlap XMM-Newton fields; we detect 14 of them in $\sim 10-50 \mathrm{ks}$ exposures. Given the difference in flux limits at $24 \mu \mathrm{m}$, the relative X-ray depths are comparable, although the higher redshifts of the Alonso-Herrero et al. and Donley et al. samples should lead to these objects being more detectable at $\mathrm{X}$-ray wavelengths due to the negative $k$-correction of an absorbed $\mathrm{X}$-ray spectrum.

One final point of interest is that the three highest redshift objects in our sample are all obscured quasars. Although the statistics are small, the fact that these objects were found in spite of strong selection effects in favor of lower redshift objects is an indication that at very high redshifts $(z \gtrsim 3)$ obscured accretion may be even more common.

\section{CONCLUSIONS}

The results presented in this paper, taken together with studies of higher redshift objects selected from mid-infrared deep fields and objects found using joint mid-infrared-radio selection, show that a large population of luminous obscured AGNs exists at $z \gtrsim 0.3$, including a significant population with bolometric luminosities above the Seyfert-quasar divide. We find that the obscured population dominates in number density over the unobscured population over a wide range in AGN luminosity, from Seyfert galaxies to luminous quasars. Comparison with AlonsoHerrero et al. (2006) suggests little variation in the obscured-tounobscured ratio with redshift for AGNs with strong mid-infrared emission.

Our results contrast with X-ray studies, which typically find that obscured AGNs are confined to low redshifts $(z \lesssim 1)$ and luminosities below the Seyfert-quasar divide, $L_{\mathrm{X}} \lesssim 10^{44} \mathrm{ergs} \mathrm{s}^{-1}$ (e.g., Trump et al. 2007), suggesting that high-luminosity and high-redshift obscured quasars are missing from current X-raybased surveys, presumably due to limitations of areal coverage and depth. Although objects with columns $N_{\mathrm{H}} \sim 10^{23} \mathrm{~cm}^{2}$ can be found in deep X-ray surveys, locally $\sim 50 \%$ of AGNs are Compton thick, with columns $>N_{\mathrm{H}} \sim 10^{24} \mathrm{~cm}^{-2}$, and the equivalents of these objects at high redshifts would be very hard to find in the X-ray, even allowing for the positive $k$-correction. This has important implications for models of the X-ray background (e.g., Worsley et al. 2005). However, it is clear that all the techniques used to find obscured AGNs are incomplete. Mid-infrared selection presumably fails for weak AGNs, for highly obscured AGNs, and at high redshifts. Our highest redshift objects are selected not on the basis of their hot dust emission but on the basis of dustreddened accretion disk emission, so we will inevitably miss the most obscured objects at $z \gtrsim 3$.

For most objects, classification of galaxy type (AGN or starburst) based on optical emission-line ratios and classification based on mid-infrared IRAC color appear to agree fairly well. The mid-infrared criterion is seen to fail for $z \lesssim 0.3$ AGNs with strong PAH emission in the IRAC $8.0 \mu \mathrm{m}$ band from an accompanying starburst. There are also some objects with AGN-like IRAC colors that lack the high-ionization emission-line spectra of AGNs. For most of these, their nature (AGN or starburst) remains to be resolved.

The discovery of a large population of obscured AGNs has important implications for accretion efficiency and black hole de- mographics. The mass density of black holes in the universe today may be accurately estimated using the bulge-luminosity black hole mass relation, and the amount of accretion luminosity arising from unobscured accretion may be estimated from the optical quasar luminosity function (Soltan 1982). Recent estimates using this technique place the radiative efficiency of accretion close to that expected from a nonspinning (Schwarzschild) black hole (e.g., Yu \& Tremaine 2002). However, if most accretion is actually hidden, to maintain the same mass density of black holes today in the face of more accretion activity requires a higher radiative efficiency, which can only be obtained from rapidly spinning Kerr black holes.

We would like to thank Alejo Martínez-Sansigre and Steve Rawlings for obtaining the WHT spectra, Michael Gregg for obtaining the Lick spectra, and the anonymous referee for a helpful report. The SWIRE team is thanked for producing and making available their Spitzer catalogs. Most of the optical data were obtained at the Hale Telescope, Palomar Observatory, as part of a continuing collaboration between the California Institute of Technology (CIT), the Jet Propulsion Laboratory (JPL; operated by CIT for the National Aeronautics and Space Administration [NASA]), and Cornell University. M. L. and A. P. were visiting astronomers at the IRTF, which is operated by the University of Hawaii under Cooperative Agreement NCC 5-538 with NASA, Science Mission Directorate, Planetary Astronomy Program. This paper is based on observations made with the Spitzer Space Telescope, which is operated by JPL, CIT, under a NASA contract. Support for this work was provided by NASA through JPL. Funding for the creation and distribution of the SDSS Archive has been provided by the Alfred P. Sloan Foundation, the Participating Institutions, NASA, the National Science Foundation, the US Department of Energy, the Japanese Monbukagakusho, and the Max Planck Society. This research has made extensive use of the NASA/IPAC Extragalactic Database, which is operated by JPL, CIT, under contract with NASA. Some of the data presented herein were obtained at the W. M. Keck Observatory, which is operated as a scientific partnership among CIT, the University of California, and NASA. The authors wish to recognize and acknowledge the very significant cultural role and reverence that the summit of Mauna Kea has always had within the indigenous Hawaiian community.

\section{APPENDIX A}

\section{COMPARISON OF CLASSIFICATIONS WITH THOSE OF PAPOVICH ET AL.}

In an independent study, Papovich et al. (2006) obtained spectra of bright $24 \mu \mathrm{m}$ XFLS sources with the Hectospec fiber spectrograph on the MMT. Several of these objects are common to our XFLS AGN sample. Papovich et al. attempted to classify their objects using a semiautomated algorithm. For the most part, our classifications agree, and where we differ it is usually because the Papovich et al. "galaxy" classification generally includes narrow-line AGNs (Table 7). There are, however, a few objects classified as broad-line quasars or AGNs for which there seems to be no evidence for broad lines in either our spectra or the Hectospec spectra. In one case, however (SSTXFLS J171430.7+ 584225), the Hectospec spectrum does show weak, broad $\mathrm{H} \beta$ and $\mathrm{Mg}$ II $\lambda 2798$ emission, which appears very weak or absent in our Palomar COSMIC spectrum. The Hectospec spectrum is 
TABLE 7

Non-SDSS Objects in the XFLS Sample with Spectra in Papovich et al. (2006)

\begin{tabular}{|c|c|c|c|c|c|}
\hline Name & $\begin{array}{c}S_{24.0} \\
(\mathrm{mJy})\end{array}$ & Redshift & Class & Basis & P06 Class ${ }^{\mathrm{a}}$ \\
\hline SSTXFLS J171115.2+594906 …................... & 9.4 & 0.587 & Sbt & & Galaxy \\
\hline SSTXFLS J171147.4+585839 .......................... & 4.8 & 0.800 & 2 & {$[\mathrm{Ne} \mathrm{v}] 13$} & Galaxy \\
\hline SSTXFLS J171324.2+585549 .......................... & 4.9 & 0.609 & 2 & {$[\mathrm{Ne} \mathrm{v}] 12$} & Galaxy \\
\hline SSTXFLS J171325.1+590531 ………................ & 9.5 & 0.126 & 2 & BPT & $\mathrm{QSO} / \mathrm{AGN}$ \\
\hline SSTXFLS J171331.5+585804 …….................... & 5.9 & 0.435 & $\mathrm{Sbt} / \mathrm{L}$ & ... & Galaxy \\
\hline SSTXFLS J171335.1+584756 .......................... & 23.7 & 0.133 & 1R & $\mathrm{Fe}$ II & $\mathrm{QSO} / \mathrm{BL}$ \\
\hline 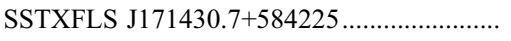 & 8.2 & 0.561 & 2 & {$[\mathrm{Ne} \mathrm{v}] 20$} & $\mathrm{QSO} / \mathrm{BL}$ \\
\hline SSTXFLS J171454.4+584948....................... & 4.9 & 0.253 & Sbt & BPT & Galaxy/star-forming \\
\hline SSTXFLS J171513.8+594638 ………................ & 5.0 & 0.248 & $1 \mathrm{R}$ & BL (radio-loud) & QSO/starburst \\
\hline SSTXFLS J171530.7+600216 & 11.6 & 0.420 & 2 & {$[\mathrm{Ne} \mathrm{v}] 26$} & $\mathrm{QSO} / \mathrm{BL}$ \\
\hline 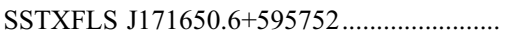 & 6.7 & 0.182 & Sbt & BPT & Galaxy/starburst \\
\hline 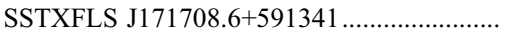 & 5.4 & 0.646 & 2 & {$[\mathrm{Ne} \mathrm{v}] 37$} & $\mathrm{QSO} / \mathrm{BL}$ \\
\hline 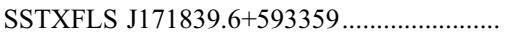 & 10.9 & 0.383 & 1 & BL (radio-loud) & $\mathrm{QSO} / \mathrm{BL}$ \\
\hline 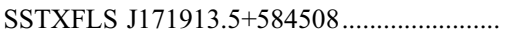 & 8.9 & 0.318 & 2 & {$[\mathrm{Ne} v] 12, \mathrm{BPT}$} & QSO \\
\hline SSTXFLS J172219.5+594506 …..................... & 7.8 & 0.271 & 2 & $\mathrm{BPT}$ & QSO/AGN_BROAD \\
\hline 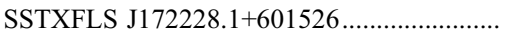 & 7.1 & 0.741 & 2 & {$[\mathrm{Ne} \mathrm{v}]$} & Galaxy/manual \\
\hline SSTXFLS J172245.0+590328 ……................... & 4.7 & 0.797 & 2 & {$[\mathrm{Ne} \mathrm{v}] 43$} & Galaxy \\
\hline
\end{tabular}

${ }^{\text {a }}$ P06 class is the classification of the object in the MMT Hectospec survey of Papovich et al. (2006), in the form class/subclass.

of higher signal-to-noise ratio than our spectrum, and the $\mathrm{H} \beta$ line falls close to the $7600 \AA$ atmospheric absorption feature (for which we do not attempt to correct), so it is most likely that we simply missed the weak, broad $\mathrm{H} \beta$ line in this case. We have therefore reclassified this object as type 1R. One further discrepancy is in our classification of SSTXFLS J171454.4+584948; this object is classified as a QSO/starburst by Papovich et al., but it plots among the starbursts on the BPT plot of Figure 4 (right) and has thus been classified as a starburst.

\section{APPENDIX B}

\section{SPECTROSCOPY OF THE $8 \mu \mathrm{m}$ FLUX-LIMITED SAMPLE}

Most of the objects in the $8 \mu \mathrm{m}$ flux-limited sample of candidate obscured AGNs of Lacy et al. (2004, 2005a) are also members of the XFLS sample of Table 3; however, a few have $24 \mu \mathrm{m}$ fluxes below $4.6 \mathrm{mJy}$ and are hence missing. For completeness we list their redshifts, where measured, and spectral types in Table 8 (see also Lacy et al. 2005a).

TABLE 8

Objects in the $8 \mu \mathrm{m}$-Selected Sample of Paper I Not in the $24 \mu \mathrm{m}$ FluX-limited XFLS Sample

\begin{tabular}{|c|c|c|c|c|}
\hline Name & $\begin{array}{c}S_{8.0} \\
(\mathrm{mJy})\end{array}$ & $z$ & Class & Basis \\
\hline SSTXFLS J171106.8+590436 & 1.38 & 0.462 & $2 ?$ & {$[\mathrm{O}$ III] $\lambda 5007 /[\mathrm{O}$ ІІ] $\lambda 3727=3.8$} \\
\hline 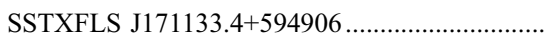 & 1.91 & $?$ & $1 ?$ & $\ldots$ \\
\hline SSTXFLS J171421.3+602239 & 1.25 & $?$ & 2/Sbt/L? & $\ldots$ \\
\hline SSTXFLS J171804.6+602705 & 1.05 & 0.43 ? & 2/Sbt/L? & $\ldots$ \\
\hline SSTXFLS J171930.9+594751 ……………......... & 1.44 & 0.358 & 2 & {$[\mathrm{Ne} v] 30$} \\
\hline SSTXFLS J172253.9+582955 & 1.00 & $1.753^{*}$ & 1 & BL \\
\hline SSTXFLS J172458.3+591545 & 1.10 & 0.494 & $2 ?$ & {$[\mathrm{O}$ III] $\lambda 5007 /[\mathrm{O}$ II $] \lambda 3727=1.8$} \\
\hline SSTXFLS J172601.8+601100 & 1.43 & $1.12 ?$ & $1 \mathrm{R}$ & Single BL \\
\hline
\end{tabular}

NoтE.-An asterisk indicates that the spectrum is from Papovich et al. (2006).

Alonso-Herrero, A., et al. 2006, ApJ, 640, 167

Antonucci, R. 1993, ARA\&A, 31, 473

Baldwin, J. A., Phillips, M. M., \& Terlevich, R. 1981, PASP, 93, 5 (BPT)

Barmby, P., et al. 2006, ApJ, 642, 126

Becker, R. H., et al. 2001, ApJS, 135, 227

Brand, K., et al. 2006, ApJ, 644, 143

Brown, M. J., et al. 2006, ApJ, 638, 88

Caccianiga, A., et al. 2004, A\&A, 416, 901

Chiappetti, L., et al. 2005, A\&A, 439, 413

Comastri, A., Setti, G., Zamorani, G., \& Hasinger, G. 1995, A\&A, 296, 1

Comastri, A., et al. 2002, ApJ, 571, 771

Cutri, R. M., Nelson, B. O., Kirkpatrick, J. D., Huchra, J. P., \& Smith, P. S. 2001, in ASP Conf. Ser. 232, The New Era of Wide Field Astronomy, ed. R. Clowes, A. Adamson, \& G. Bromage (San Francisco: ASP), 78

Donley, J. L., Rieke, G. H., Rigby, J. R., \& Pérez-Gonzales, P. G. 2005, ApJ, 634, 169

\section{REFERENCES}

Eckart, M. E., et al. 2006, ApJS, 165, 19

Fadda, D., Jannuzi, B., Ford, A., \& Storrie-Lombardi, L. J. 2004, AJ, 128, 1

Fadda, D., et al. 2006, AJ, 131, 2859

Glikman, E., Gregg, M. D., Lacy, M., Helfand, D. J., Becker, R. H., \& White, R. L. 2004, ApJ, 607, 60

Grimes, J. P., Kriss, G. A., \& Espey, B. R. 1999, ApJ, 526, 130

Haas, M., et al. 2004a, A\&A, 419, L49 2004b, A\&A, 424, 531

Hatziminaoglou, E., et al. 2005, AJ, 129, 1198

Kauffmann, G., et al. 2003, MNRAS, 346, 1055

Kewley, L. J., Groves, B., Kauffmann, G., \& Heckman, T. 2006, MNRAS, 372, 961

Lonsdale, C. J., et al. 2003, PASP, 115, 897

Lacy, M., et al. 2004, ApJS, 154, 166 (Paper I) 2005a, Mem. Soc. Astron. Italiana, 76, 154 2005b, ApJS, 161, 41 
Lawrence, A. 1991, MNRAS, 252, 586

Leipski, C., et al. 2005, A\&A, 440, L5

Martínez-Sansigre, A., et al. 2005, Nature, 436, 666 2006, MNRAS, 370, 1479

Mason, R. E., et al. 2006, ApJ, 640, 612

Meisenheimer, K., Haas, M., Müller, S. A. H., Chini, R., Klaas, U., \& Lemke, D. 2001, A\&A, 372, 719

Michel, L., Herent, O., Motch, C., Pye, J., \& Watson, M. G. 2004, in ASP Conf. Ser. 314, Astronomical Data Analysis Software and Systems XIII, ed. M. Oschenbein, M. Allen, \& D. Egret (San Francisco: ASP), 570

Moran, E. C., Filippenko, A. V., \& Chornock, R. 2002, ApJ, 579, L71

Ogle, P., Whysong, D., \& Antonucci, R. 2006, ApJ, 647, 161

Papovich, C., et al. 2006, AJ, 132, 231

Pei, Y. C. 1992, ApJ, 395, 130

Polletta, M., et al. 2006, ApJ, 642, 673

Rayner, J. T., et al. 2003, PASP, 115, 362

Richards, G. T., et al. 2006, ApJS, 166, 470

Rigby, J. R., Rieke, G. H., Donley, J. L., Alonso-Herrero, A., \& Pérez-González, P. G. 2006, ApJ, 645, 115

Sajina, A., Lacy, M., \& Scott, D. 2005, ApJ, 621, 256
Sharp, R. G., Sabbey, C. N., Vivas, A. K., Oemler, A., McMahon, R. G., Hodgkin, S. T., \& Coppi, P. S. 2002, MNRAS, 337, 1153

Siana, B., et al. 2007, in Infrared Diagnostics of Galaxy Evolution, ed. R. Chary, in press (astro-ph/0604373)

Soltan, A. 1982, MNRAS, 200, 115

Spinoglio, L., Malkan, M. A., Rush, B., Carrasco, L., \& Recillas-Cruz, E. 1995, ApJ, 453, 616

Stern, D., et al. 2005, ApJ, 631, 163

Treister, E., et al. 2004, ApJ, 616, 123

Trump, J. R., et al. 2007, ApJS, in press (astro-ph/0606016)

Vanden Berk, D. E., et al. 2001, AJ, 122, 549

van Ojik, R., Röttgering, H. J. A., Miley, G. K., Bremer, M. N., Maccetto, F., \& Chambers, K. C. 1994, A\&A, 289, 54

Watson, M. G., et al. 2004, BAAS, 36, 1202

Weedman, D., Le Floch, E., Higdon, S. J. U., Higdon, J. L., \& Houck, J. R. 2006, ApJ, 638, 613

Worsley, M. A., et al. 2005, MNRAS, 357, 1281

Yu, Q., \& Tremaine, S. 2002, MNRAS, 335, 965

Yuan, F., \& Narayan, R. 2004, ApJ, 612, 724

Zakamska, N. L., et al. 2003, AJ, 126, 2125 